\title{
Thin accretion disks around cold Bose-Einstein condensate stars
}

\author{
Bogdan Dănilă ${ }^{1, a}$, Tiberiu Harko ${ }^{2, b}$, Zoltán Kovács ${ }^{3, c}$ \\ ${ }^{1}$ Department of Physics, Babes-Bolyai University, Kogalniceanu Street, Cluj-Napoca, Romania \\ 2 Department of Mathematics, University College London, Gower Street, London WC1E 6BT, UK \\ ${ }^{3}$ Max-Fiedler-Str. 7, 45128 Essen, Germany
}

Received: 19 March 2015 / Accepted: 22 April 2015 / Published online: 9 May 2015

(C) The Author(s) 2015. This article is published with open access at Springerlink.com

\begin{abstract}
Due to their superfluid properties some compact astrophysical objects, like neutron or quark stars, may contain a significant part of their matter in the form of a BoseEinstein condensate (BEC). Observationally distinguishing between neutron/quark stars and BEC stars is a major challenge for this latter theoretical model. An observational possibility of indirectly distinguishing BEC stars from neutron/quark stars is through the study of the thin accretion disks around compact general relativistic objects. In the present paper, we perform a detailed comparative study of the electromagnetic and thermodynamic properties of the thin accretion disks around rapidly rotating BEC stars, neutron stars and quark stars, respectively. Due to the differences in the exterior geometry, the thermodynamic and electromagnetic properties of the disks (energy flux, temperature distribution, equilibrium radiation spectrum, and efficiency of energy conversion) are different for these classes of compact objects. Hence in this preliminary study we have pointed out some astrophysical signatures that may allow one to observationally discriminate between BEC stars and neutron/quark stars.
\end{abstract}

\section{Introduction}

Since its proposal by Bose [1] and the generalization by Einstein $[2,3]$, the quantum statistics of integer spin particles (bosons) did represent a fundamental field of study in both theoretical and experimental physics. One of the most important properties of bosonic systems is their phase transition to a condensed state, in which all particles are in the same quantum ground state. This quantum bosonic system is called a Bose-Einstein condensate (BEC), and from a physical point of view it is characterized by a sharp peak over a broader distribution in both coordinate and momentum space. The quan-

\footnotetext{
a e-mail: bogdan.danila22@gmail.com

be-mail: t.harko@ucl.ac.uk

c e-mail: kovacsz2013@yahoo.com
}

tum explanation for this behavior is that in a BEC particles become correlated with each other, and their wavelengths overlap. Note that the correlation means that the thermal wavelength $\lambda_{\mathrm{T}}$ is greater than the mean inter-particle distance $l$. It occurs at a critical temperature $T_{\mathrm{c}}<2 \pi \hbar^{2} n^{2 / 3} / m k_{\mathrm{B}}$, where $m$ is the mass of an individual condensate particle, $n$ is the number density, and $k_{\mathrm{B}}$ is Boltzmann's constant [4-11]. A coherent quantum state in the bosonic system develops either when the particle density $\rho$ is high enough, or when the temperature $T$ is sufficiently low. From the experimental point of view, the Bose-Einstein condensation can be detected by the observation of a sharp peak in the velocity distribution, which always appears when the system is below the critical temperature, $T<T_{\mathrm{c}}$.

In the laboratory the Bose-Einstein condensation was observed first in 1995 in dilute alkali gases, such as vapors of rubidium and sodium. To obtain the condensation the gases were confined in a magnetic trap and cooled down to very low temperatures [12-14]. The experiment producing a laboratory BEC did represent a major achievement in experimental condensed matter physics, and the confirmation of the old and important predictions in theoretical statistical physics of Bose and Einstein [12-14]. Note that in recent years quantum degenerate gases have been created by a multitude of experimental methods, including combination of laser and evaporative cooling techniques. Thus the observation of the Bose-Einstein condensation did open several new lines of multidisciplinary research at the border of atomic, statistical and condensed matter physics [4-14].

The Bose-Einstein condensation processes are assumed to play an important role in the understanding of many fundamental processes in condensed matter physics. For example, superfluidity of low temperature liquids, like ${ }^{3} \mathrm{He}$, can be explained by assuming a Bose-Einstein condensation process [8]. It is interesting to note that experimental observations as well as quantum theoretical calculations estimate the condensate fraction $n_{0}$ at $T=0$ for superfluid helium to be 
only around $n_{0} \approx 0.10$, a very small amount. Hence, since a strongly correlated pair of fermions behaves approximately like a boson, the arising liquid helium superfluidity can be interpreted as resulting from the Bose-Einstein condensation of coupled fermions. A very similar physical model can be used to describe the transition to a superconducting state in a solid material (metal). Superconductivity can thus be interpreted as the condensation of electrons (or holes) to Cooper pairs. The formation of the Cooper pairs drastically reduces the resistance caused by the motion of electrons in metals, and thus it leads to the formation of a superconductor [8].

Since Bose-Einstein condensation is a phenomenon that has been observed and thoroughly studied in terrestrial laboratories, the possibility that it may also occur in bosonic systems existing on astrophysical or cosmological scales cannot be rejected a priori. Thus, it was proposed that dark matter, which is required to explain the dynamics of the neutral hydrogen clouds at large distances from the centers of the galaxies, and which is assumed to be a cold bosonic gravitationally bounded system, could also exist in the form of a BEC [15-20]. A systematic study of the properties of BoseEinstein condensed galactic dark matter halos was initiated in [21], and the astrophysical and cosmological implications of the existence of Bose-Einstein condensed dark matter have been investigated in detail recently [22-50].

From a theoretical point of view it was shown in [21] that by introducing the Madelung representation of the wave function, the dynamics of the BEC dark matter halo is described by the continuity equation, and the hydrodynamic Euler equations of the standard classical fluid mechanics. Hence, Bose-Einstein condensed dark matter can be described theoretically as a gas, with the pressure and density related by a barotropic equation of state (EOS). In the case of a self-interacting condensate dark matter with quartic self-interaction potential, the EOS is polytropic with index $n=1$ [21].

Bose-Einstein condensation could play an important role in nuclear and quark matter physics, in the framework of the so-called Bardeen-Cooper-Schrieffer (BCS) to BEC crossover. From a theoretical point of view it is expected that at ultra-high densities nuclear matter exists in the form of a degenerate Fermi gas of quarks. The Cooper pairs of quarks form near the Fermi surface a BEC. Hence high density nuclear matter represents, from a physical point of view, a so-called color superconductor $[51,52]$. When the attractive interaction between fermions is strong enough, and the temperature drops below the critical temperature, the fermions condense to the bosonic zero mode, and form a quark BEC [53-63]. As a first step toward the formation of the BEC the fermions must form a BCS state, which can be realized when the attractive interaction between particles is weak. This system exhibits superfluid properties, which are characterized by the existence of an energy gap for single-particle exci- tations. The energy gap is created by the formation of the Cooper pairs. On the other hand a BEC is formed when the attractive interaction between fermions is extremely strong. This interaction first leads to the formation of bound particles (bosons), which at some critical temperature $T_{\mathrm{c}}$ start to condense to the bosonic zero mode. It is important to mention that the BCS and BEC states are smoothly connected (crossover), without a phase transition between the two phases [64-68]. For a recent review of the BCS-BEC crossover see [69].

The possibility of the existence of some forms of BECs in neutron stars has been considered a long time ago (see [70] for a detailed discussion). One possibility for the formation of a BEC in a dense neutron star is the condensation of the negatively charged mesons, leading to the replacement of electrons with very high Fermi momenta by mesons [70]. Bose-Einstein condensation of kaons and anti-kaons in compact objects was also investigated in detail [71,72]. It turns out that the presence of pion or kaon condensates may have at least two important effects on the global properties of dense neutron stars. Firstly, Bose-Einstein condensation softens the EOS of the stellar matter above the critical density for the onset of condensation. An important consequence of the softening of the EOS is the reduction of the maximum neutron star mass. On the other hand, due to the softening of the EOS, the central density increases significantly. Secondly, the condensation of mesons would lead to considerably enhanced neutrino luminosities, much higher than those of normal neutron matter. The increase in neutrino luminosity has important consequences on the neutron star cooling [70]. Another particle, which may be present inside neutron stars, and which may form a BEC, is the H-dibaryon. The $\mathrm{H}$-dibaryon is a doubly strange six-quark composite. It has zero spin and isospin, and a baryon number $B=2$ [70]. Neutron star matter may also contain an important fraction of $\Lambda$ hyperons, neutral subatomic hadrons. They consist of one up, one down and one strange quark, and they are labeled $\Lambda^{0}[73,74]$. The $\Lambda$ hyperons may also combine to form $\mathrm{H}$ dibaryons [75-77]. Thus, H-matter BECs may also exist at the center of very dense neutron stars, where the matter density is extremely high [70]. An interesting possibility is that neutrino superfluidity, as suggested by Kapusta [78], may also lead to Bose-Einstein condensation inside neutron stars [79].

Thus theoretical results in nuclear matter physics indicate that the possibility of the existence of some forms of Bose-Einstein condensed matter inside compact astrophysical objects, like neutron or quark stars, or even the existence of stars formed entirely from a pure BEC, cannot be excluded a priori. The properties of pure BEC stars have been considered in [80]. It was shown that stars formed of BECs with particle masses of the order of two neutron masses (Cooper pair) and scattering length of the order of 10-20 fm have maximum masses of the order of $2 M_{\odot}$, maximum central 
densities of the order of $0.1-0.3 \times 10^{16} \mathrm{~g} / \mathrm{cm}^{3}$, and minimum radii in the range of 10-20 km. Hence Bose-Einstein condensed stars can form a large class of stable astrophysical objects, whose basic astrophysical parameters (mass and radius) sensitively depend on the mass of the condensed particle and on the scattering length. It was also suggested that the recently observed neutron stars with masses in the range of 2-2.4M $\odot$ (Vela X-1, 4U 1700-377, and the black widow pulsar B1957+20) are BEC stars. Further properties of BEC stars have been considered in [81-84].

The structure of static and rotating BEC stars was investigated numerically in [85] by solving the Gross-PitaevskiiPoisson system of coupled differential equations. It was shown, with longer simulation runs, that within the computational limits of the simulation the BEC stars are stable. The physical properties of the self-gravitating BEC were investigated in both non-rotating and rotating cases.

A fundamental theoretical problem is to find some clear astrophysical signatures, going beyond the global stellar parameters (mass and radius) that could indicate the presence of a BEC inside a neutron star, or to give a firm observational evidence for the existence of pure BEC stars. It is the purpose of the present paper to suggest that such a specific signature, indicating the presence of a pure BEC star, may indeed exist, and it can be obtained from the study of the radiation emission of thin accretion disks that usually form around compact general relativistic objects.

The growth of most astrophysical objects is determined by mass accretion. Due to the presence of interstellar matter, accretion disks are generally formed around compact objects. Accretion disks are well known observationally, representing flattened astronomical structures. They are made of rapidly rotating hot gas, slowly spiraling onto a central massive and dense object. The gravitational energy of the gas motion is a source of heat, generated by the internal stresses and the dynamical friction. A small fraction of the heat is converted into radiation, which partially escapes. The radiation emission cools down the accretion disk. Therefore important information about the accretion disk physics comes from the radiation emitted from the disk. The radiation, detected in the radio, optical or X-ray frequency bands, allows astronomers to analyze its electromagnetic spectrum and its time variability. Thus essential results as regards the physics of the disks can be obtained from observations.

The cooling of the disk via the electromagnetic radiation emission from its surface represents an efficient mechanism that prevents the extreme heating of the disk. On the other hand, the thermodynamic equilibrium established in this way allows the disk to stabilize its thin vertical size. Usually the inner edge of the thin disk is located at the marginally stable orbit of the compact object potential. Hence in higher orbits the hot gas has a Keplerian motion [86,87]. Since the electromagnetic radiation emission from the thin disk is determined by the external gravitational potentials, which in turn are determined by the EOS of the dense neutron or quark matter in the star, astrophysical observations of the emission spectra from accretion disks may lead to the possibility of directly testing the EOS of the dense matter inside compact general relativistic objects.

The emissivity properties of the accretion disks have been used to investigated, and obtain distinctive astrophysical signatures, for large classes of compact astrophysical objects, including naked singularities [88], gravastars [89], and wormholes [90,91]. Specific electromagnetic disk signatures in different modified gravity theories, such as $f(R)$ gravity, brane world models, Chern-Simons models and the Horava-Lifshitz theory were considered [92-96], while the properties of accretion disks around rotating and non-rotating neutron, quark, boson or fermion stars have been analyzed in [97-104].

In the present paper we perform a comparative study of the rotational properties and of the disk emission properties for five neutron star EOSs, and of the quark matter bag model EOS with the polytropic $n=1$ BEC EOS. The main goal of this preliminary investigation is to point out the possible existence of some observational signatures that may distinguish between these different classes of compact objects. We begin our analysis by considering the global rotational properties of the considered compact objects. To obtain the equilibrium configurations of the rotating neutron, quark, and BEC stars we use the rotating neutron star (RNS) code, as introduced in [105,106], and discussed in detail in [107]. The RNS software provides the metric potentials for different types of equations of state of compact rotating general relativistic objects. We investigate three different cases, corresponding to stellar models with fixed mass and angular velocity, stellar models rotating at Keplerian frequencies, and stellar models with fixed central density and fixed polar radius to equatorial radius ratio. As a next step in our study we use the exterior metrics to obtain the physical properties of the accretion disks for the considered equations of state. Particular signatures appear in the electromagnetic spectrum of the BEC stars, thus leading to the theoretical possibility of directly testing, and discriminating, the BEC EOS of the dense matter by using astrophysical observations of the emission spectra from accretion disks.

The present paper is organized as follows. In Sect. 2 we briefly review the basics of the Bose-Einstein condensation and discuss the properties of Newtonian BEC stars. The electromagnetic and thermodynamic properties of accretion disks around compact general relativistic objects are described in Sect. 3. The equations of state of the neutron, quark, and BEC matter, as well as the global astrophysical properties of the considered stellar models are obtained and discussed in Sect. 4. The electromagnetic and thermodynamic properties (flux, luminosity, and temperature distribution) of the accre- 
tion disks around neutron, quark, and BEC stars are obtained in Sect. 5. We discuss our results and conclude our study in Sect. 6.

\section{Bose-Einstein condensation}

The most important characteristic of a quantum system of $N$ interacting bosons in the condensed state is that most of the particles lie in the same single-particle quantum state. For a bosonic quantum system consisting of an extremely large number of particles, the calculation of the physical parameters of the ground state with the direct use of the Hamiltonian is generally very difficult, due to the high computational cost. A significant simplification of the many-body type computations can be achieved with the use of some approximate methods. One such approximate semi-analytic method is the mean field analysis of the quantum condensate. The basic idea of the mean field method is the separation of the BEC contribution to the total bosonic field operator. In the following in our analysis we assume that the bosonic astrophysical system consists of scalar (zero spin) particles with non-zero mass. From a simplifying physical point of view we assume that when the system makes a transition to a Bose-Einstein condensed phase, the range of Van der Waals-type scalar mediated interactions among particles becomes infinite.

\subsection{The Gross-Pitaevskii equation}

In the second quantization approach the Hamiltonian describing a many-body system of interacting bosons, confined by an external potential $V_{\text {ext }}$, is given by

$$
\begin{aligned}
\hat{H}= & \int \mathrm{d} \vec{r} \hat{\Phi}^{+}(\vec{r})\left[-\frac{\hbar^{2}}{2 m} \nabla^{2}+V_{\text {rot }}(\vec{r})+V_{\text {ext }}(\vec{r})\right] \hat{\Phi}(\vec{r}) \\
& +\frac{1}{2} \int \mathrm{d} \vec{r} \mathrm{~d} \vec{r}^{\prime} \hat{\Phi}^{+}(\vec{r}) \hat{\Phi}^{+}\left(\vec{r}^{\prime}\right) V\left(\vec{r}-\vec{r}^{\prime}\right) \hat{\Phi}(\vec{r}) \hat{\Phi}\left(\vec{r}^{\prime}\right),
\end{aligned}
$$

where $\hat{\Phi}(\vec{r})$ and $\hat{\Phi}^{+}(\vec{r})$ are the bosonic field operators, and $V\left(\vec{r}-\vec{r}^{\prime}\right)$ is the two-body interatomic potential, respectively [4-11]. The bosonic field operators annihilate and create a particle at the position $\vec{r}$. $V_{\text {rot }}(\vec{r})$ is the potential associated to the rotation of the condensate.

In order to obtain a significant simplification of the mathematical formalism a number of approximate methods have been developed. One such approximate and simplifying semi-analytic approach is the mean field description of the condensate. In this approach the condensate contribution to the bosonic field operator is separated out. For a uniform gas confined in a volume $V$, BEC occurs in the single particle state $\Phi_{0}=1 \sqrt{V}$, having zero momentum. The field operator can then be decomposed in the form
$\hat{\Phi}(\vec{r})=\sqrt{N / V}+\hat{\Phi}^{\prime}(\vec{r})$. By treating the operator $\hat{\Phi}^{\prime}(\vec{r})$ as a small perturbation, the first order theory for the excitations of the interacting Bose gases can be fully developed [4-11].

In the general case of a non-uniform and time-dependent configuration the Heisenberg representation of the field operator is given by

$$
\hat{\Phi}(\vec{r}, t)=\psi(\vec{r}, t)+\hat{\Phi}^{\prime}(\vec{r}, t),
$$

where $\psi(\vec{r}, t)$, also called the condensate wave function, is the expectation value of the field operator, $\psi(\vec{r}, t)=$ $\langle\hat{\Phi}(\vec{r}, t)\rangle$. It is important to note that the wave function $\psi(\vec{r}, t)$ is a classical field, and its absolute value fixes the particle number density of the condensate through the relation $\rho(\vec{r}, t)=|\psi(\vec{r}, t)|^{2}$. On the other hand the normalization condition for the condensate wave function is $N=\int \rho(\vec{r}, t) d^{3} \vec{r}$, where $N$ is the total number of particles in the BEC.

The condensate wave function satisfies the following equation of motion, which can be obtained from Heisenberg equation corresponding to the many-body Hamiltonian given by Eq. (1):

$$
\begin{aligned}
& i \hbar \frac{\partial}{\partial t} \hat{\Phi}(\vec{r}, t)=[\hat{\Phi}, \hat{H}]=\left[-\frac{\hbar^{2}}{2 m} \nabla^{2}+V_{\text {rot }}(\vec{r})+V_{\text {ext }}(\vec{r})\right. \\
& \left.+\int \mathrm{d} \vec{r}^{\prime} \hat{\Phi}^{+}\left(\vec{r}^{\prime}, t\right) V\left(\vec{r}^{\prime}-\vec{r}\right) \hat{\Phi}\left(\vec{r}^{\prime}, t\right)\right] \hat{\Phi}(\vec{r}, t)
\end{aligned}
$$

The zeroth-order approximation to the Heisenberg equation is obtained by replacing $\hat{\Phi}(\vec{r}, t)$ with the condensate wave function $\psi$. For short distances this is in general a poor approximation for computing the integral containing the particle-particle interaction $V\left(\vec{r}^{\prime}-\vec{r}\right)$. However, it is important to note that in a dilute and cold gas, at low energy only binary collisions are important. These collisions can be characterized, independently of the exact form of the two-body potential, by a single physical parameter, the swave scattering length $a$. Therefore, one can obtain a very good approximation by replacing the generally unknown potential $V\left(\vec{r}^{\prime}-\vec{r}\right)$ with an effective interaction potential $V\left(\vec{r}^{\prime}-\vec{r}\right)=\lambda \delta\left(\vec{r}^{\prime}-\vec{r}\right)$, with the coupling constant $\lambda$ determined by the scattering length $l_{a}$ via the relation $\lambda=4 \pi \hbar^{2} a / m$, where $m$ is the mass of the condensate particles. With the use of the effective potential the integral in the bracket of Eq. (3) can easily be calculated to give $\lambda|\psi(\vec{r}, t)|^{2}$. Hence the resulting approximate equation of motion for the condensate is the Schrödinger equation with a quartic nonlinear term [4-11], also called the GrossPitaevskii equation. However, in order to obtain a more general and realistic description of the BEC stars, one may also assume an arbitrary nonlinear self-interaction term of the form $g\left(|\psi(\vec{r}, t)|^{2}\right)=g(\rho)[4]$.

Therefore the non-relativistic generalized Gross-Pitaevskii equation, describing a gravitationally trapped rotating 
$\mathrm{BEC}$, is given by

$$
\begin{aligned}
i \hbar \frac{\partial}{\partial t} \psi(\vec{r}, t)= & {\left[-\frac{\hbar^{2}}{2 m} \nabla^{2}+V_{\mathrm{rot}}(\vec{r})+V_{\mathrm{ext}}(\vec{r})\right.} \\
& \left.+g^{\prime}\left(|\psi(\vec{r}, t)|^{2}\right)\right] \psi(\vec{r}, t)
\end{aligned}
$$

where we have denoted $g^{\prime}=\mathrm{d} g / \mathrm{d} \rho$. As for $V_{\text {ext }}(\vec{r})$, we assume that it is the gravitational potential, denoted for simplicity by $V, V_{\text {ext }}=V$. The gravitational potential satisfies the Poisson equation of Newtonian gravity,

$\nabla^{2} V=4 \pi G \rho_{m}$,

where $\rho_{m}=m \rho=m|\psi(\vec{r}, t)|^{2}$ is the mass density inside the BEC.

\subsection{The hydrodynamical representation}

The physical as well as the astrophysical the properties of a BEC, described by the generalized Gross-Pitaevskii equation Eq. (4) can be analyzed, computed, and understood much more easily by using the so-called Madelung representation of the wave function [4]. In this representation we write the condensate wave function $\psi$ in the form

$\psi(\vec{r}, t)=\sqrt{\rho(\vec{r}, t)} \exp \left[\frac{i}{\hbar} S(\vec{r}, t)\right]$

where the function $S(\vec{r}, t)$ has the physical dimensions of an action. By substituting the above expression of $\psi(\vec{r}, t)$ into Eq. (4), it follows that the generalized Gross-Pitaevskii equation decouples into a system of two first order partial differential equations for the two real functions $\rho_{m}$ and $\vec{v}$, respectively, given by

$$
\begin{aligned}
\frac{\partial \rho_{m}}{\partial t}+\nabla \cdot\left(\rho_{m} \vec{v}\right)=0, & \\
\rho_{m}\left[\frac{\partial \vec{v}}{\partial t}+(\vec{v} \cdot \nabla) \vec{v}\right]= & -\nabla P\left(\frac{\rho_{m}}{m}\right)-\rho_{m} \nabla\left(\frac{V_{\mathrm{rot}}}{m}\right) \\
& -\rho_{m} \nabla\left(\frac{V_{\mathrm{ext}}}{m}\right)-\nabla V_{\mathrm{Q}},
\end{aligned}
$$

where we have introduced the quantum potential $V_{\mathrm{Q}}$, defined as

$V_{\mathrm{Q}}=-\frac{\hbar^{2}}{2 m} \frac{\nabla^{2} \sqrt{\rho_{m}}}{\sqrt{\rho_{m}}}$,

the velocity of the Bose-Einstein condensed fluid $\vec{v}$, given by

$\vec{v}=\frac{\nabla S}{m}$ and we have denoted

$P\left(\frac{\rho_{m}}{m}\right)=g^{\prime}\left(\frac{\rho_{m}}{m}\right) \frac{\rho_{m}}{m}-g\left(\frac{\rho_{m}}{m}\right)$.

From its definition we can immediately see that the velocity field $\vec{v}$ is irrotational. Thus it satisfies the condition $\nabla \times \vec{v}=0$. Therefore we obtain the important result that in the Madelung representation the equations of motion of the BEC in a gravitational field take the form of the equation of continuity and of the hydrodynamic Euler equations, respectively. Hence a BEC in an external gravitational field can be described as a gas whose density and pressure are related by a barotropic EOS [4-11]. The exact form of the EOS of the BEC depends on the nonlinearity term $g$.

In the important case of a gravitationally bounded BEC with a very large number of particles the quantum pressure is important only near the external boundary of the quantum system. Hence in all astrophysically important situations the quantum pressure term is much smaller than the nonlinear interaction term [21].

In the most studied approach to the BECs, the nonlinearity term $g$ is quadratic and is given by

$g\left(\rho_{m}\right)=\frac{u_{0}}{2}|\psi|^{4}=\frac{u_{0}}{2} \rho_{m}^{2}$,

where $u_{0}=4 \pi \hbar^{2} l_{a} / m$ [4-11]. The corresponding EOS of the condensate is

$P(\rho)=K \rho^{2}$.

Therefore we have obtained the very important result that the EOS of the standard BEC with quartic nonlinearity is a polytrope, with index $n=1$.

In the following for simplicity we consider only the case of the Bose-Einstein condensates with quartic nonlinearity. In this particular case the physical properties of the condensates are also relatively well-known from numerous laboratory experiments. From a theoretical point of view it is important to note that their properties can be described in terms of only two free parameters, the mass $m$ of the condensate particle, and the scattering length $a$, describing the particle interaction, respectively.

\subsection{Masses and radii of Newtonian static BEC stars}

Since the EOS of a cold BEC star is a polytrope of index $n=1$, all the physical properties of the star can be derived from the well-known Lane-Emden equation, given by [80]

$\frac{1}{\xi^{2}} \frac{\mathrm{d}}{\mathrm{d} \xi}\left(\xi^{2} \frac{\mathrm{d} \theta}{\mathrm{d} \xi}\right)=-\theta$, 
where $\theta$ is a dimensionless variable defined via $\rho=\rho_{c} \theta$, with $\rho_{r} m c$ the central density of the star, and $\xi$ is a dimensionless radial coordinate. The general non-singular solution of Eq. (14) is well known and can be represented as $\theta(\xi)=\sin \xi / \xi$. We define the dimensionless radius of the star by the condition $\theta\left(\xi_{1}\right)=0$, giving $\xi_{1}=\pi$. Hence the physical radius $R$ of the BEC star can be represented as a function of the scattering length and the fundamental physical constants as [80]

$R=\pi \sqrt{\frac{\hbar^{2} a}{G m^{3}}}=6.61\left(\frac{a}{1 \mathrm{fm}}\right)^{1 / 2}\left(\frac{m}{2 m_{n}}\right)^{-3 / 2} \mathrm{~km}$.

For $m=2 m_{n}$ and $a=1 \mathrm{fm}$ the numerical value of the radius of the BEC star is $R \approx 7 \mathrm{~km}$. It is interesting to note that the radius of the gravitationally bounded pure BEC star is independent on the central density and on the mass of the star and is fully determined by the physical characteristics $a$ and $m$ of the condensate only.

The mass of the BEC star can be found as [80]

$M=4 \pi^{2}\left(\frac{\hbar^{2} a}{G m^{3}}\right)^{3 / 2} \rho_{c}$,

or, equivalently,

$M=1.84\left(\frac{\rho_{c}}{10^{16} \mathrm{~g} \mathrm{~cm}^{-3}}\right)\left(\frac{a}{1 \mathrm{fm}}\right)^{3 / 2}\left(\frac{m}{2 m_{n}}\right)^{-9 / 2} M_{\odot}$.

For $m=2 m_{n}, a=1 \mathrm{fm}$, and $\rho_{r} m c=5 \times 10^{15} \mathrm{~g} / \mathrm{cm}^{3}$, the mass of the condensate is $M \approx 0.92 M_{\odot}$. On the other hand by taking for the mass of the condensate particle the numerical value $m=m_{\mathrm{K}}^{*}=m_{n} / 10$, where $m_{\mathrm{K}}^{*}$ is the kaon mass, we obtain an extremely high maximum mass of $M=63.50 M_{\odot}$ for the kaon condensate star, with a corresponding radius of $R=355 \mathrm{~km}$. Note that the mass of the static condensate can be expressed in terms of the radius and central density by

$M=\frac{4}{\pi} \rho_{c} R^{3}$

The above important equation shows that the mean density of the $\operatorname{star} \bar{\rho}=3 M / 4 \pi R^{3}$ can be obtained from the central density of the condensate by the simple relation $\bar{\rho}=3 \rho_{c} / \pi^{2}$.

Under a scaling of the parameters $m, a$, and $\rho_{c}$ so that $m \rightarrow \alpha_{1} m, a \rightarrow \alpha_{2} a, \rho_{c} \rightarrow \alpha_{3} \rho_{c}$, the radii and the masses of the Newtonian BEC stars have the scaling properties [80],

$R \rightarrow \alpha_{1}^{-3 / 2} \alpha_{2}^{1 / 2} R, \quad M \rightarrow \alpha_{1}^{-9 / 2} \alpha_{2}^{3 / 2} \alpha_{3} M$

It is important to point out that general relativistic effects impose strong constraints on the structure and global param- eters of the BEC stars, and that the values obtained by the simple Newtonian estimates may highly exceed the stability limit imposed by the general relativistic analysis.

\section{Electromagnetic radiation properties of thin accretion disks in stationary axisymmetric space-times}

In order to investigate the accretion disk properties around neutron, quark, and BEC stars we first briefly introduce the general formalism that allows us to describe the electromagnetic radiation properties of thin accretion disks in stationary axisymmetric space-times. In our presentation we closely follow the approach developed in [97]. As a first step in our study we consider the basic kinematic properties of massive particles moving in stable circular orbits in stationary and axially symmetric geometries. The general formalism for obtaining the constants of the motion (energy and angular momentum), the effective gravitational potential and the radii of the marginally stable orbits are presented in some detail. Then we briefly review the basic equations describing the electromagnetic radiation emission from accretion disks, and we present the general expressions for the flux, temperature distribution, and luminosity of the disk.

\subsection{Massive particle motion in stationary and axially symmetric space-times}

In this work our main emphasis is on the analysis of the physical properties and characteristics of particles that form a thin accretion disk, and move in circular stable orbits around general relativistic compact objects. The exterior geometry created by a central dense object is assumed to be stationary and axially symmetric, given in full generality by the following metric:

$$
\mathrm{d} s^{2}=g_{t t} \mathrm{~d} t^{2}+2 g_{t \phi} \mathrm{d} t \mathrm{~d} \phi+g_{r r} \mathrm{~d} r^{2}+g_{\theta \theta} \mathrm{d} \theta^{2}+g_{\phi \phi} \mathrm{d} \phi^{2} .
$$

In the equatorial approximation, i.e., $|\theta-\pi| \ll 1$, which we adopt in our study, all the metric functions $g_{t t}, g_{t \phi}, g_{r r}, g_{\theta \theta}$, and $g_{\phi \phi}$ depend on the radial coordinate $r$ only $[86,87]$. In the following we denote the square root of the determinant of the metric tensor by $\sqrt{-g}$.

In order to determine the electromagnetic properties of the disk we first obtain the radial dependence of the angular velocity $\Omega$, of the specific energy $\widetilde{E}$ and of the specific angular momentum $\widetilde{L}$ for particles moving in circular orbits around compact objects in the geometry given by Eq. (20). All these physical parameters can be obtained from the geodesic equations, which for the considered metric take the following form [88-96]: 


$$
\begin{aligned}
& \frac{\mathrm{d} t}{\mathrm{~d} s}=\frac{\widetilde{E} g_{\phi \phi}+\widetilde{L} g_{t \phi}}{g_{t \phi}^{2}-g_{t t} g_{\phi \phi}}, \\
& \frac{\mathrm{d} \phi}{\mathrm{d} s}=-\frac{\widetilde{E} g_{t \phi}+\widetilde{L} g_{t t}}{g_{t \phi}^{2}-g_{t t} g_{\phi \phi}}, \\
& g_{r r}\left(\frac{\mathrm{d} r}{\mathrm{~d} s}\right)^{2}=-1+\frac{\widetilde{E}^{2} g_{\phi \phi}+2 \widetilde{E} \widetilde{L} g_{t \phi}+\widetilde{L}^{2} g_{t t}}{g_{t \phi}^{2}-g_{t t} g_{\phi \phi}} .
\end{aligned}
$$

We define an effective potential term $V_{\text {eff }}(r)$ as

$V_{\mathrm{eff}}(r)=-1+\frac{\widetilde{E}^{2} g_{\phi \phi}+2 \widetilde{E} \widetilde{L} g_{t \phi}+\widetilde{L}^{2} g_{t t}}{g_{t \phi}^{2}-g_{t t} g_{\phi \phi}}$.

For particles moving in stable circular orbits in the equatorial plane the potential $V_{\text {eff }}(r)$ must satisfy the following two important conditions: $V_{\text {eff }}(r)=0$ and $V_{\text {eff, }}(r)=0$, respectively, where a comma denotes the derivative with respect to the radial coordinate $r$. From these conditions we obtain the specific energy, the specific angular momentum, and the angular velocity of particles moving in circular orbits for the case of spinning general relativistic compact objects in the form [97]

$$
\begin{aligned}
\widetilde{E} & =-\frac{g_{t t}+g_{t \phi} \Omega}{\sqrt{-g_{t t}-2 g_{t \phi} \Omega-g_{\phi \phi} \Omega^{2}}}, \\
\widetilde{L} & =\frac{g_{t \phi}+g_{\phi \phi} \Omega}{\sqrt{-g_{t t}-2 g_{t \phi} \Omega-g_{\phi \phi} \Omega^{2}}}, \\
\Omega & =\frac{\mathrm{d} \phi}{\mathrm{d} t}=\frac{-g_{t \phi, r}+\sqrt{\left(g_{t \phi, r}\right)^{2}-g_{t t, r} g_{\phi \phi, r}}}{g_{\phi \phi, r}} .
\end{aligned}
$$

The marginally stable orbit around the central object can be determined from the supplementary condition $V_{\text {eff, } r r}(r)=$ 0 , which provides the following important relationship:

$\widetilde{E}^{2} g_{\phi \phi, r r}+2 \widetilde{E} \widetilde{L} g_{t \phi, r r}+\widetilde{L}^{2} g_{t t, r r}-\left(g_{t \phi}^{2}-g_{t t} g_{\phi \phi}\right)_{, r r}=0$.

Once the metric coefficients $g_{t t}, g_{t \phi}$, and $g_{\phi \phi}$ are explicitly given, by inserting Eqs. (25)-(27) into Eq. (28), and numerically solving this equation for the radial coordinate $r$, we obtain the radii of the marginally stable orbits of massive particles in stable circular orbits around a rotating general relativistic high density compact object [88-97].

\subsection{Electromagnetic emissivity of thin accretion disks}

In the following we concentrate on thin accretion disks, that is, accretion disks having their vertical size negligible as compared to their horizontal extensions. This means that the disk height $H$, which we define as the maximum half thickness of the disk in the vertical direction, is much smaller than the characteristic radius $R$ of the disk, $H \ll R$. We further assume that the thin disk is in hydrodynamical equilibrium. Moreover, we fully neglect the possible effects of the pressure gradient, and of the vertical entropy gradient in the disk. The heat generated by internal stresses and dynamical friction is emitted over the disk surface, and this emission prevents the disk from heating up at extremely high temperatures. On the other hand, this equilibrium determines the disk to stabilize its thin vertical size. An important astrophysical parameter, the inner edge of the thin disk, is located at the marginally stable orbit of the central object gravitational potential. Hence the accreting matter has a Keplerian motion in higher orbits.

In the steady-state accretion disk model, the mass accretion rate $\dot{M}_{0}$ is a constant that does not change in time. An effective physical description of the disk properties is obtained by averaging all the quantities describing the orbiting gas over a characteristic time scale, e.g. $\Delta t$, over the azimuthal angle $\Delta \phi=2 \pi$, and over the height $H$, respectively $[86,108,109]$.

As we have already seen, the particles move in a Keplerian orbit around the dense compact object with the four-velocity $u^{\mu}$, have a rotational velocity $\Omega=\mathrm{d} \phi / \mathrm{d} t$, a specific energy $\widetilde{E}$, and a specific angular momentum $\widetilde{L}$. In the steady-state thin disk model all these quantities depend only on the radii $r$ of the orbits. The particles form a disk of an averaged surface density $\Sigma$, which is obtained as the average of the rest mass density $\rho_{0}$ of the gas in the vertical direction. The matter in the disk is described by an anisotropic fluid source. The density $\rho_{0}$ of the disk, the energy flow vector $q^{\mu}$, and the dissipative part of the energy-momentum tensor $t^{\mu \nu}$ are all measured in the averaged rest-frame. In this specific frame the specific heat and heat transfer processes are neglected. Then one important parameter characterizing the disk structure is the surface density of the disk [86,109],

$\Sigma(r)=\int_{-H}^{H}\left\langle\rho_{0}\right\rangle \mathrm{d} z$

which is obtained as the averaged rest mass density $\left\langle\rho_{0}\right\rangle$ over $\Delta t$ and $2 \pi$. Another important disk parameter is the torque

$W_{\phi}^{r}=\int_{-H}^{H}\left\langle t_{\phi}^{r}\right\rangle \mathrm{d} z$

representing the average of the component $\left\langle t_{\phi}^{r}\right\rangle$ of the energymomentum tensor over $\Delta t$ and $2 \pi$. The time and orbital average of the energy flux vector gives the radiation flux $F(r)$ from the disk surface as

$F(r)=\left\langle q^{z}\right\rangle$.

The energy-momentum tensor of the matter in the disk is represented in its standard form according to

$T^{\mu \nu}=\rho_{0} u^{\mu} u^{\nu}+2 u^{(\mu} q^{\nu)}+t^{\mu \nu}$, 
where the four-velocity of the matter satisfies the conditions $u_{\mu} q^{\mu}=0, u_{\mu} t^{\mu \nu}=0$. The four-vectors of the energy and angular momentum flux are defined by $-E^{\mu} \equiv T_{\nu}^{\mu}(\partial / \partial t)^{v}$ and $J^{\mu} \equiv T_{\nu}^{\mu}(\partial / \partial \phi)^{\nu}$, respectively. Note that the structure equations of the thin disk can be derived by integrating the conservation laws of the rest mass, of the energy, and of the angular momentum of the gas forming the disk, respectively $[86,109]$. From the equation of the rest mass conservation, $\nabla_{\mu}\left(\rho_{0} u^{\mu}\right)=0$, first the important result follows that the time averaged mass accretion rate is independent of the disk radius,

$\dot{M}_{0} \equiv-2 \pi \sqrt{-g} \Sigma u^{r}=$ constant.

The conservation law $\nabla_{\mu} E^{\mu}=0$ of the energy can be reformulated in integral form, more interesting from a physical point of view, as [97]

$\left[\dot{M}_{0} \widetilde{E}-2 \pi \sqrt{-g} \Omega W_{\phi}^{r}\right]_{, r}=4 \pi \sqrt{-g} F \widetilde{E}$.

The above equation shows that the energy transported by the mass flow, $\dot{M}_{0} \widetilde{E}$, and the energy transported by the dynamical stresses in the disk, $2 \pi \sqrt{-g} \Omega W_{\phi}{ }^{r}$, is exactly compensated by the energy radiated away from the surface of the disk, $4 \pi \sqrt{-g} F \widetilde{E}$. The law of the angular momentum conservation, $\nabla_{\mu} J^{\mu}=0$, indicates the equilibrium between the three forms of the angular momentum transport,

$\left[\dot{M}_{0} \widetilde{L}-2 \pi r W_{\phi}^{r}\right]_{, r}=4 \pi \sqrt{-g} F \widetilde{L}$.

By eliminating $W_{\phi}{ }^{r}$ from Eqs. (34) and (35), with the use of the universal energy-angular momentum relation $\mathrm{d} E=$ $\Omega \mathrm{d} J$ for circular geodesic orbits, written in the form $\widetilde{E}_{, r}=$ $\Omega \widetilde{L}_{, r}$, the flux $F$ of the electromagnetic energy emitted by the disk surface is obtained as $[86,97,109]$

$F(r)=-\frac{\dot{M}_{0}}{4 \pi \sqrt{-g}} \frac{\Omega_{, r}}{(\widetilde{E}-\Omega \widetilde{L})^{2}} \int_{r_{\text {in }}}^{r}(\widetilde{E}-\Omega \widetilde{L}) \widetilde{L}_{, r} \mathrm{~d} r$,

where $r_{\text {in }}$ is the inner edge of the disk. In our study we assume that $r_{\text {in }}=r_{\mathrm{ms}}$. Note that the flux depends of the specific energy, angular momentum, and angular velocity of the gas motion around the central general relativistic compact object.

The gas forming the accretion disk in the steady-state thin disk model is assumed to be in thermodynamical equilibrium. Therefore the radiation emitted by the disk surface may be considered as perfect black body radiation, depending on the temperature only, with the energy flux given by

$F(r)=\sigma_{\mathrm{SB}} T^{4}(r)$,

where $\sigma_{\mathrm{SB}}$ is the Stefan-Boltzmann constant. Once the radiative flux is known, from Eq. (37) we obtain the temperature distribution on the disk surface.
The observed luminosity $L(v)$ of the disk surface has a red-shifted black body spectrum, given by [98]

$$
\begin{aligned}
L(v) & =4 \pi \mathrm{d}^{2} I(v) \\
& =\frac{8 \pi h \cos \gamma}{c^{2}} \int_{r_{\text {in }}}^{r_{f}} \int_{0}^{2 \pi} \frac{v_{\mathrm{e}}^{3} r \mathrm{~d} \phi \mathrm{d} r}{\exp \left(h \nu_{\mathrm{e}} / k_{B} T\right)-1} .
\end{aligned}
$$

In Eq. (38) $h$ is Planck's constant, $k_{B}$ is Boltzmmann's constant, $d$ is the distance to the source (disk), $I(v)$ is the Planck distribution function, $v_{\mathrm{e}}$ is the frequency of the emitted radiation, $\gamma$ is the disk inclination angle, and $r_{\text {in }}$ and $r_{f}$ indicate the positions of the inner and outer edge of the disk, respectively. In the natural system of units with $\hbar=c=k_{\mathrm{B}}=1$, we obtain for the disk luminosity the expression

$L(v)=16 \pi^{2} \cos \gamma \int_{r_{\text {in }}}^{r_{f}} \int_{0}^{2 \pi} \frac{v_{\mathrm{e}}^{3} r \mathrm{~d} \phi \mathrm{d} r}{\exp \left(2 \pi v_{\mathrm{e}} / T\right)-1}$.

In the following we take the upper limit of integration in Eq. (38) as infinity, that is, we assume $r_{f} \rightarrow \infty$. Moreover, we expect that the flux from the disk surface vanishes at $r \rightarrow \infty$. This condition is independent on the geometry of the general relativistic compact object. The frequency of the emitted radiation is given by the relation $v_{\mathrm{e}}=v(1+z)$, where the red-shift factor can be written as

$$
1+z=\frac{1+\Omega r \sin \phi \sin \gamma}{\sqrt{-g_{t t}-2 \Omega g_{t \phi}-\Omega^{2} g_{\phi \phi}}} .
$$

In Eq. (40) we have neglected the light bending [110-112]. This approximation works well for small inclination angles, but it is not so good for large inclination angles (edge-on disks).

Another important characteristics of the mass accretion processes is the efficiency $\epsilon$ with which the central object converts the mass of the gas into radiation. The efficiency $\epsilon$ is defined as the ratio of the rate of the energy of photons emitted from the disk surface, and the rate at which mass-energy is transported to the central high density general relativistic object $[86,109]$. Both energies are measured at infinity. If all photons escape to infinity, the efficiency depends on the specific energy measured at the marginally stable orbit $r_{\mathrm{ms}}$ only,

$\epsilon=1-\widetilde{E}_{\mathrm{ms}}$.

For Schwarzschild black holes the efficiency $\epsilon$ is about $6 \%$. This result is independent on whether the photon capture by the black hole is considered, or not. For rapidly rotating black holes for which the capture of radiation by the black hole is ignored, $\epsilon$ is equal to $42 \%$. For a Kerr black hole with the photon capture explicitly considered the efficiency is $40 \%$ [87]. 
Note that the fluxes and the emission spectra of the accretion disks around compact objects satisfy some simple, but important, scaling relations. Such scaling relations can be found when one considers the scaling transformation of the radial coordinate, given by $r \rightarrow \widetilde{r}=r / M$, where $M$ is the mass of the central object. Under such a scaling transformation in general the metric tensor coefficients are invariant, while the specific energy, the angular momentum and the angular velocity transform as $\widetilde{E} \rightarrow \widetilde{E}$, $\widetilde{L} \rightarrow M \widetilde{L}$, and $\Omega \rightarrow \widetilde{\Omega} / M$, respectively. The flux scales as $F(r) \rightarrow F(\widetilde{r}) / M^{4}$, giving the simple scaling law of the temperature as $T(r) \rightarrow T(\widetilde{r}) / M$. Another important rescaling is the transformation of the frequency of the emitted radiation as $v \rightarrow \widetilde{v}=v / M$. Under this rescaling of the radiation frequency the luminosity of the disk scales as $L(v) \rightarrow L(\widetilde{v}) / M$ [97]. On the other hand, since the flux is proportional to the accretion rate $\dot{M}_{0}$, we obtain the important result that an increase in the accretion rate leads to a linear increase of the radiation flux from the disk.

From an observational point of view, the use of the thermal radiation from thin disks to test the nature of a compact general relativistic object is often called the continuum-fitting method, and was proposed in [113] to investigate the observational consequences of black hole spin in X-ray binaries. The standard thin accretion disk model introduced in [86] was used to analyze the emissivity of accretion disks around black holes. In the case of Kerr black holes the observed disk spectrum, however, bears several important corrections to the simple formula given by Eq. (36). Since the X-rays are emitted on the hot inner disk, in this region electron scattering may dominate over the free-free absorption. As a consequence the color temperature may be greater than the effective temperature, with the inner disk radiating like a diluted black body. The general relativistic corrections near the event horizon (gravitational red-shift and focusing) can cause the observed color temperature and the integrated flux to be different from the true local values. The continuumfitting method was extensively used to observationally study the spin of black holes [114-117].

\section{Equations of state and physical parameters of the neutron, quark, and BEC stars}

To set the stage for our study, in the present section we introduce first the equations of state of the dense nuclear matter considered in our comparative study of the neutron, quark, and BEC stars, and present some of the basic dynamical properties of the corresponding rotating stellar models. In order to compare the properties of the BEC stars with other compact objects we choose five equations of state of neutron matter, and the bag model EOS for quark matter. We restrict our study to rotating BEC stars satisfying the $n=1$ polytropic
EOS. The astrophysical parameters of the stars described by the adopted equations of state are also presented in detail. In order to compare the properties of the stellar models we consider three classes of models. In the first model we fix the values of the mass and of the angular velocity for all stars. As a second case we consider compact stars rotating at the maximum (Keplerian) frequencies. Finally, as a third class of models we consider stars having the same central density and fixed ratio of the equatorial and polar radii, respectively.

\subsection{Equations of state of the neutron, quark, and BEC matter}

In order to obtain a consistent and realistic physical description of the rapidly rotating general relativistic compact stars, as a first step we have to adopt the equations of state for the dense neutron, quark, and BEC stellar matter, respectively. In the present comparative study of the physical properties of the accretion disks as regards rapidly rotating compact general relativistic objects we adopt the following equations of state [97]:

1. Akmal-Pandharipande-Ravenhall (APR) [118] EOS. As a first example of an EOS of dense neutron matter we consider EOS APR, which was obtained numerically by using the variational chain summation methods and the Argonne $v_{18}$ two-nucleon interaction, respectively. Note that boost corrections to the two-nucleon interaction, giving the leading relativistic effect of order $(v / c)^{2}$, as well as three-nucleon interactions, are also included in the basic nuclear Hamiltonian. The dense matter density range described by EOS APR is from $2 \times 10^{14}$ to $2.6 \times 10^{15} \mathrm{~g} / \mathrm{cm}^{3}$. The maximum mass limit in the static case for this EOS is $2.20 M_{\odot}$. To obtain a full description of the neutron star properties the APR EOS is joined to the composite Baym-Bethe-Pethick (BBP) $\left(\varepsilon / c^{2}>4.3 \times 10^{11} \mathrm{~g} / \mathrm{cm}^{3}\right)$ [119]-Baym-PethickSutherland $\left(10^{4}<4.3 \times 10^{11} \mathrm{~g} / \mathrm{cm}^{3}\right)[120]-$ FeynmanMetropolis-Teller $\left(\varepsilon / c^{2}<10^{4} \mathrm{~g} / \mathrm{cm}^{3}\right)$ [121] equations of state, respectively.

2. Douchin-Haensel (DH) [122] EOS. EOS DH represents a complete EOS of the neutron star matter. It describes both the neutron star crust and its liquid core. It is constructed by using the effective nuclear interaction SLy of the Skyrme type. The corresponding interaction potential describes very well the properties of very rich neutron matter. On the other hand the structure of the crust and its EOS are obtained in the zero temperature approximation only, and under the assumption of the ground state composition. The EOS of the liquid core is calculated assuming (minimal) npe $\mu$ composition. The density range is from $3.49 \times 10^{11}$ to $4.04 \times 10^{15} \mathrm{~g} / \mathrm{cm}^{3}$. The minimum 
and maximum masses of the static neutron stars for the DH EOS are $0.094 M_{\odot}$ and $2.05 M_{\odot}$, respectively.

3. Shen-Toki-Oyamatsu-Sumiyoshi (STOS) [123] EOS. The STOS EOS of nuclear matter is obtained by using the relativistic mean field (RMF) theory with nonlinear $\sigma$ and $\omega$ terms. It can be used in a wide neutron matter density and temperature range, with various proton fractions. The EOS was specifically designed for the use of supernova explosion simulation and for the calculations of the neutron star properties. To compute the properties of inhomogeneous nuclear matter, where heavy nuclei are formed together with free nucleon gas, the ThomasFermi approximation is used. In the present paper we consider only the zero temperature STOS EOS, namely the EOS for $T=0$, and we denote it as STOSO.

4. RMF equations of state with isovector scalar mean field in the presence of the $\delta$-meson field- RMF soft and RMF stiff EOSs [124]. Even that in symmetric nuclear matter the $\delta$-meson mean field vanishes, this field still can significantly influence the properties of asymmetric nuclear matter in neutron stars. Note that the RMF contribution due to the $\delta$-field to the nuclear symmetry energy is smaller than zero. Therefore the energy per particle of neutron matter is larger at high densities, as compared to the case when no $\delta$-field included. Also, the proton fraction of $\beta$-stable matter increases. Splitting of proton and neutron effective masses due to the $\delta$-field may significantly affect the transport properties of neutron star matter. These equations of state can be parameterized by the coupling parameters $C_{\sigma}^{2}=g_{\sigma}^{2} / m_{\sigma}^{2}, C_{\omega}^{2}=g_{\omega}^{2} / m_{\omega}^{2}$, $\bar{b}=b / g_{\sigma}^{3}$, and $\bar{c}=c / g_{\sigma}^{4}$, respectively, where $m_{\sigma}$ and $m_{\omega}$ are the masses of the respective mesons, and $b$ and $c$ are the coefficients in the potential energy $U(\sigma)$ of the $\sigma$-field. The soft RMF EOS is parameterized by $C_{\sigma}^{2}=1.582 \mathrm{fm}^{2}, C_{\omega}^{2}=1.019 \mathrm{fm}^{2}, \bar{b}=-0.7188$, and $\bar{c}=6.563$, while the stiff RMF EOS is parameterized by $C_{\sigma}^{2}=11.25 \mathrm{fm}^{2}, C_{\omega}^{2}=6.483 \mathrm{fm}^{2}, \bar{b}=0.003825$, and $\bar{c}=3.5 \times 10^{-6}$, respectively.

5. Baldo-Bombaci-Burgio (BBB) [125] EOS. The BBB EOS is an EOS for asymmetric nuclear matter. It is derived from the Brueckner-Bethe-Goldstone manybody theory with explicit three-body forces taken into account. Two EOSs are obtained, one corresponding to the Argonne AV14 (BBBAV14), and the other to the Paris two-body nuclear force (BBBParis), implemented by the Urbana model for the three-body force. The maximum static mass configurations are $M_{\max }=1.8 M_{\odot}$ and $M_{\text {max }}=1.94 M_{\odot}$ when the AV14 and Paris interactions are used, respectively. The onset of direct Urca processes occurs at particle number densities $n \geq 0.65 \mathrm{fm}^{-3}$ for the AV14 potential, and $n \geq 0.54 \mathrm{fm}^{-3}$ for the Paris potential. The comparison with other microscopic models for the EOS shows noticeable differences, which can also influence significantly the neutron star properties. The density range for this EOS is from $1.35 \times 10^{14}$ to $3.507 \times 10^{15} \mathrm{~g} / \mathrm{cm}^{3}$.

6. Bag model EOS for quark matter-Q EOS [126-129]. For the description of the quark matter we adopt a simple phenomenological description, based on the MIT bag model EOS. Hence we assume that in quark matter the pressure $p$ is related to the energy density $\rho$ by

$p=\frac{1}{3}(\rho-4 B) c^{2}$,

where the parameter $B$, called the bag constant, is the difference between the energy density of the perturbative and non-perturbative quantum chromodynamic vacuum. For the bag constant we adopt the numerical value $B=$ $4.2 \times 10^{14} \mathrm{~g} / \mathrm{cm}^{3}[126,127,129]$.

7. The BEC EOS. For the BEC stars we adopt the $n=1$ polytropic EOS, given by

$P(\rho)=K \rho^{2}$,

with

$K=\frac{2 \pi \hbar^{2} a}{m^{3}}=0.1856 \times 10^{5}\left(\frac{a}{1 \mathrm{fm}}\right)\left(\frac{m}{2 m_{n}}\right)^{-3}$,

where $m_{n}=1.6749 \times 10^{-24} \mathrm{~g}$ is the mass of the neutron. The behavior of the BEC EOS, as well as of the corresponding stellar models essentially depends on the ratio $a / m^{3}$ of the scattering length and of the condensate particle mass. In the present study we restrict our analysis to three values of the ratio $(a / 1 \mathrm{fm})\left(m / 2 m_{n}\right)^{-3}$ : $(a / 1 \mathrm{fm})\left(m / 2 m_{n}\right)^{-3}=15,(a / 1 \mathrm{fm})\left(m / 2 m_{n}\right)^{-3}=$ 30 , and $(a / 1 \mathrm{fm})\left(m / 2 m_{n}\right)^{-3}=50$, respectively. We denote the corresponding equations of state of the BEC matter by BEC15, BEC30, and BEC50, respectively.

The variation of the considered equations of state with respect to the density of the dense stellar matter is represented in Fig. 1.

The low density behavior of the BEC15 and BEC30 EOSs is significantly different as compared to the other considered equations of state, indicating the possibility of the existence of stable BEC stars at pressures lower than the neutron matter pressure. In the adopted range of densities the BEC EOSs show a linear pressure-density dependence.

4.2 Astrophysical parameters of the neutron, quark, and BEC stars

In quasi-isotropic coordinates the metric outside a rotating compact general relativistic star can be represented as $[105,106]$ 


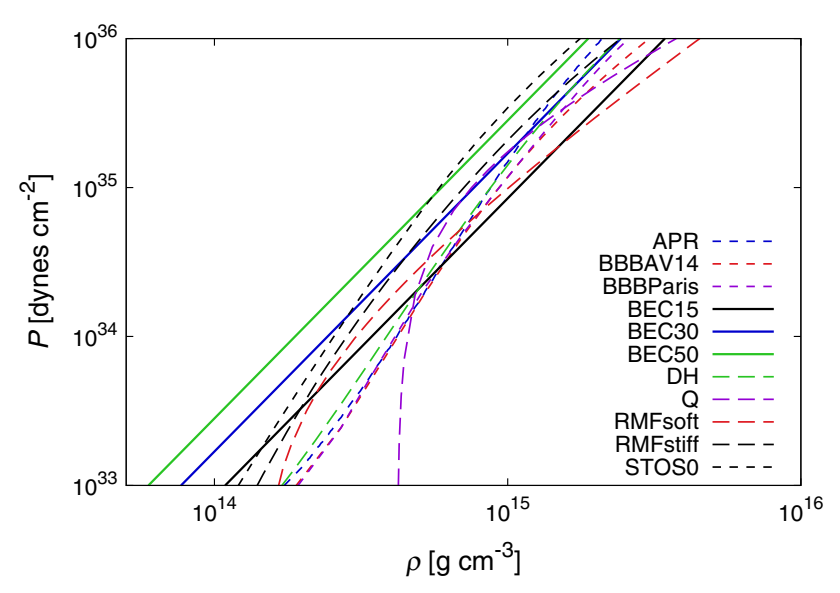

Fig. 1 Variation of the pressure as a function of the density for the considered equations of state of the neutron, quark and BEC dense matter

$$
\begin{aligned}
\mathrm{d} s^{2}= & -e^{\bar{\gamma}+\bar{\rho}} \mathrm{d} t^{2}+e^{2 \bar{\alpha}}\left(\mathrm{d} \bar{r}^{2}+\bar{r}^{2} \mathrm{~d} \theta^{2}\right)+e^{\bar{\gamma}-\bar{\rho}} \bar{r}^{2} \sin ^{2} \theta \\
& \times(\mathrm{d} \phi-\bar{\omega} \mathrm{d} t)^{2},
\end{aligned}
$$

where the metric potentials $\bar{\gamma}, \bar{\rho}, \bar{\alpha}$, and the angular velocity of the stellar fluid $\bar{\omega}$, measured relative to the local inertial frame, are all functions of the quasi-isotropic radial coordinate $\bar{r}$, and of the polar angle $\theta$. The RNS code computes numerically the metric functions in a quasi-spheroidal coordinate system, as functions of the parameter $s=\bar{r} /\left(\bar{r}+\bar{r}_{\mathrm{e}}\right)$, where $\bar{r}_{\mathrm{e}}$ is the equatorial radius of the star, which we have converted into Schwarzschild-type coordinates $r$ according to the equation $r=\bar{r} \exp [(\bar{\gamma}-\bar{\rho}) / 2]$. To obtain the radius of the marginally (or innermost) stable circular orbits $r_{\mathrm{ms}}$ we use a truncated form of the analytical approximation given as [130]

$$
\begin{aligned}
\frac{r_{\mathrm{ms}}}{6 M} \approx & 1-0.54433 q-0.22619 q^{2}+0.17989 Q_{2} \\
& -0.23002 q^{3}+0.26296 q Q_{2}-0.29693 q^{4} \\
& +0.44546 q^{2} Q_{2},
\end{aligned}
$$

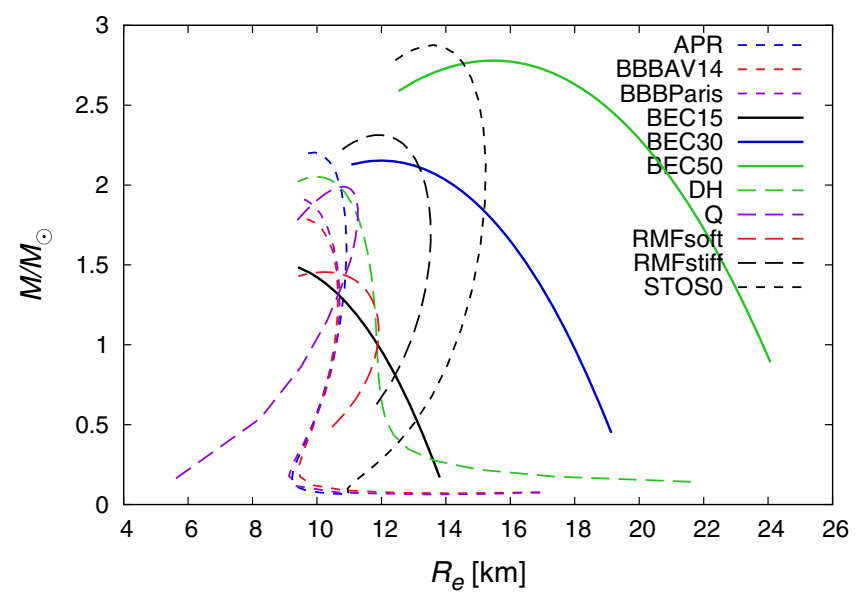

where $q=J / M^{2}$ and $Q_{2}=-M_{2} / M^{3}$, respectively, and where $J$ is the spin angular momentum, and $M_{2}$ is the quadrupole moment.

\subsubsection{Mass-radius relation for neutron, quark, and BEC stars}

The mass-radius relation of the compact general objects for the considered equations of state of the dense nuclear matter, $M=M\left(R_{\mathrm{e}}\right)$, where $R_{\mathrm{e}}$ is the circumferential radius at the equator, are presented, for four fixed values of the ratio $r_{\mathrm{p}} / r_{\mathrm{e}}$, where $r_{\mathrm{p}}$ is the polar radius, and $r_{\mathrm{e}}$ is the equatorial radius of the star, in Figs. 2 and 3, respectively.

As one can see from the figures, the BEC stars form a distinct class of stellar objects, as compared to the group of "standard" neutron and quark stars. The mass-radius relation is systematically shifted to the right region of the $M=M\left(R_{\mathrm{e}}\right)$ relation, has a specific shape, and indicates a much larger radius for the BEC star. The radius increases with increasing $a / m^{3}$, so that for $(a / 1 \mathrm{fm})\left(m / 2 m_{n}\right)^{-3}=50$ and $r_{\mathrm{p}} / r_{\mathrm{e}}=0.7$ the radius of the maximum mass stable BEC star is of the order of $30 \mathrm{~km}$. Thus a first distinctive signature of rapidly rotating BEC stars is their bigger radius, as compared to the "standard" neutron and quark stars, indicating a large value of the coefficient $a / m^{3}$. On the other hand, for $(a / 1 \mathrm{fm})\left(m / 2 m_{n}\right)^{-3}<20$, the radius of the maximum mass stable BEC star is of the same order as the radii of the "standard" stars, $R_{\mathrm{e}} \approx 8-15 \mathrm{~km}$. Significant differences appear in the maximum masses of the stars, with the maximum allowable mass of the BEC50 star varying between $2.8 M_{\odot}$ (static case) and $3.0 M_{\odot}$, for the rapidly rotating star with $r_{\mathrm{p}} / r_{\mathrm{e}}=0.7$. For the BEC15 star, the maximum mass of the stable configuration ranges between $1.5 M_{\odot}$ (static case), and $1.6 M_{\odot}$, for $r_{\mathrm{p}} / r_{\mathrm{e}}=0.7$. Hence a specific mass-radius relation provides a first distinctive feature of the BEC stars, as compared to the considered neutron and quark stars.

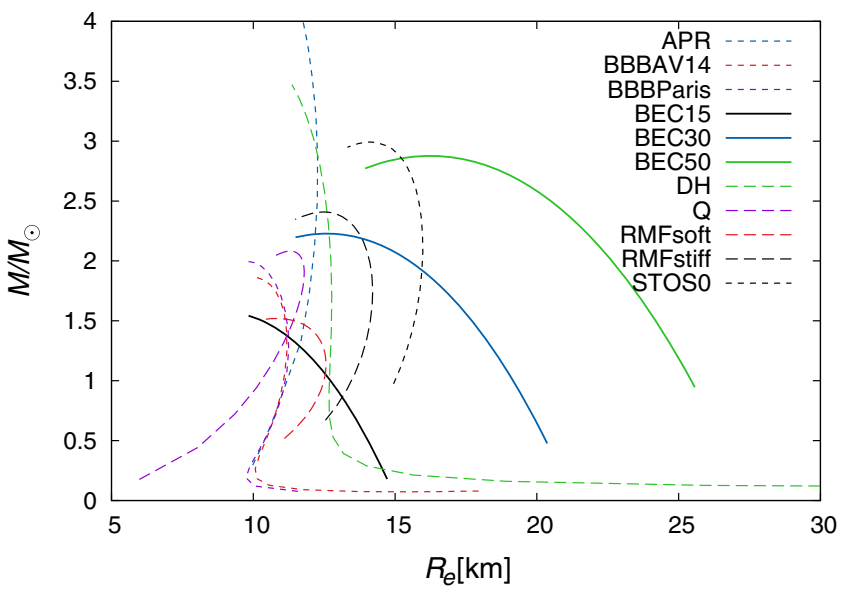

Fig. 2 Mass-circumferential radius relation for the rotating neutron, quark, and BEC stars for $r_{\mathrm{p}} / r_{\mathrm{e}}=1$ (left figure) and $r_{\mathrm{p}} / r_{\mathrm{e}}=0.9($ right figure) 

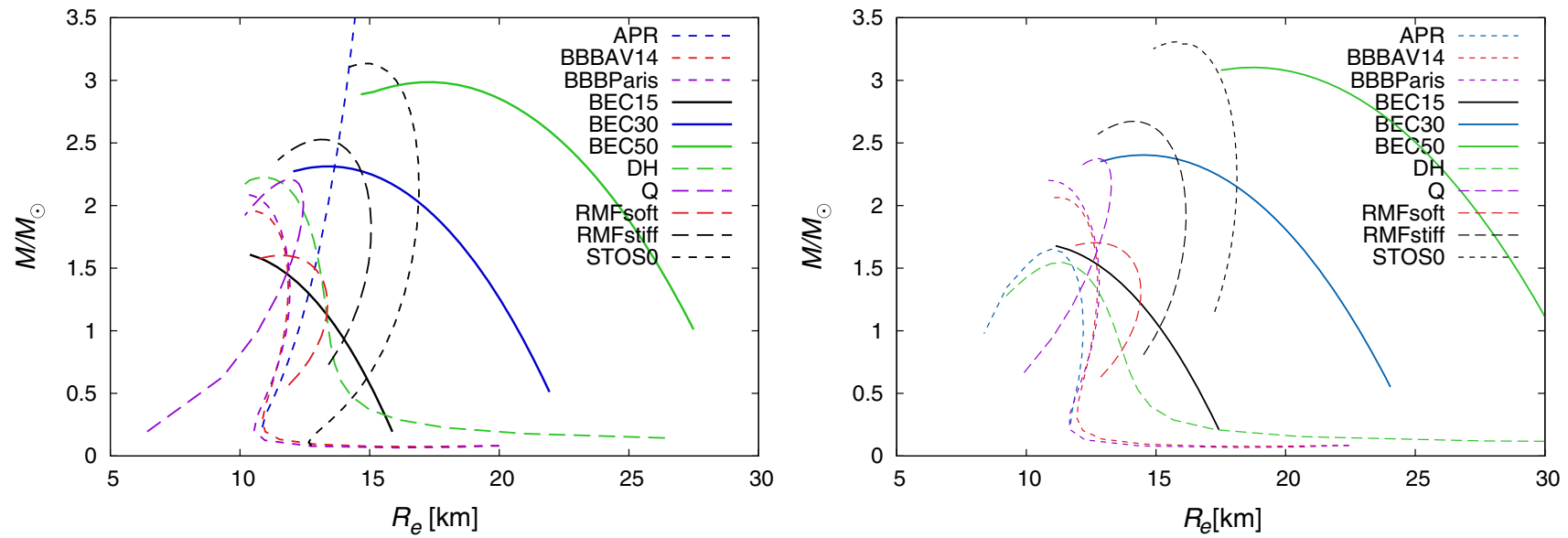

Fig. 3 Mass-circumferential radius relation for the rotating neutron, quark, and BEC stars for $r_{\mathrm{p}} / r_{\mathrm{e}}=0.8($ left figure $)$ and $r_{\mathrm{p}} / r_{\mathrm{e}}=0.7($ right figure)

\subsubsection{Models with fixed mass and angular velocity}

In analyzing the emissivity properties of the accretion disks we consider three types of stellar models, whose main astrophysical properties are presented in the following in a tabular form. In all Tables, $\rho_{r} m c$ is the central density, $M$ is the gravitational mass, $M_{0}$ is the rest mass, $R_{\mathrm{e}}$ is the circumferential radius at the equator, $\Omega$ is the angular velocity, $\Omega_{\mathrm{p}}$ is the angular velocity of a particle in circular orbit at the equator, $T / W$ is the rotational-gravitational energy ratio, $c J / G M_{\odot}^{2}$ is the angular momentum, $I$ is the moment of inertia, $\Phi_{2}$ gives the mass quadrupole moment $M_{2}$ so that $M_{2}=c^{4} \Phi_{2} /\left(G^{2} M^{3} M_{\odot}^{3}\right), h_{+}$is the height from the surface of the last stable co-rotating circular orbit in the equatorial plane, $h_{-}$is the height from surface of the last stable counterrotating circular orbit in the equatorial plane, $\omega_{r} m c / \Omega$ is the ratio of the central value of the potential $\omega$ to $\Omega, r_{\mathrm{e}}$ is the coordinate equatorial radius, and $r_{\mathrm{p}} / r_{\mathrm{e}}$ is the ratio of the axes (polar to equatorial), respectively.

The physical properties of the neutron, quark, and BEC stars with fixed mass, $M \approx 1.8 M_{\odot}$ and angular velocity $\Omega \approx 5 \times 10^{3} \mathrm{~s}^{-1}$ are presented in Table 1 .

The BEC15 EOS does not allow stellar masses of the order of $1.8 M_{\odot}$. The mass corresponding to an angular velocity of $\Omega \approx 5 \times 10^{3} \mathrm{~s}^{-1}$ is $1.541 \mathrm{M}_{\odot}$, with a small equatorial radius of the order of $6 \mathrm{~km}$, and a high central density $\rho_{r} m c=5 \times 10^{15} \mathrm{~g} / \mathrm{cm}^{3}$. Configurations with $1.8 M_{\odot}$ can be obtained for the BEC30 and BEC50 EOSs. They have equatorial radii of the order of 22 and $28 \mathrm{~km}$. A similar, but still smaller equatorial radius, $r_{\mathrm{e}}=18 \mathrm{~km}$, can be found only for the STOS0 EOS. The BEC30, BEC50, and STOS0 configurations have similar central densities. The BEC30 and BEC50 stars have the highest moment of inertia $I$, and highest angular momentum per unit $c J / G M$, with EOS STOS0 having the closest values of these parameters.

\subsubsection{Models rotating at Keplerian frequencies}

As a second astrophysical model we consider the case of the neutron, quark, and BEC stars rotating at Keplerian frequencies. The physical properties of this class of stars are presented in Table 2.

The most massive stable star for this model, with mass $M \approx 3.5 M_{\odot}$ is obtained for the STOS0 EOS. The BEC50 star has a comparable, but somewhat lower mass, of around $3.2 M_{\odot}$. The RMFstiff and the quark EOS $Q$ have Keplerian masses of the order of $2.8 M_{\odot}$, greater than the Keplerian mass of the BEC15 EOS, $M=2.31 M_{\odot}$. The smallest Keplerian mass is obtained for the BEC15 EOS. The BEC stars have the biggest equatorial radii, ranging between 15 and 17 $\mathrm{km}$. For the neutron and quark star EOSs the highest equatorial radius is obtained for the STOSO EOS, but which is still smaller than the equatorial radius of the BEC15 model. The equatorial radius of the RMSsoft EOS almost coincides with the Keplerian equatorial radius of the BEC15 EOS, with the RMSsoft EOS having a much greater mass than the BEC15 EOS one. The Keplerian model of the STOSO EOS has the greatest moment of inertia, and angular momentum per unit mass, with the BEC15 having the smallest values for these quantities.

\subsubsection{Models with fixed central density and $r_{\mathrm{p}} / r_{\mathrm{e}}=0.85$}

The astrophysical parameters of the neutron, quark, and BEC stars with a fixed central density $\rho_{r} m c=10^{15} \mathrm{~g} / \mathrm{cm}^{3}$, and a fixed ratio of the polar to the equatorial radius $r_{\mathrm{p}} / r_{\mathrm{e}}$ are presented in Table 3.

Similarly to the previous cases, the higher mass for stars having fixed central density and a polar to equatorial radius ratio $r_{\mathrm{p}} / r_{\mathrm{e}}=0.85$ is obtained for the STOS0 EOS, $M=$ $2.9792 M_{\odot}$. The mass of the BEC50 EOS model is lower, 

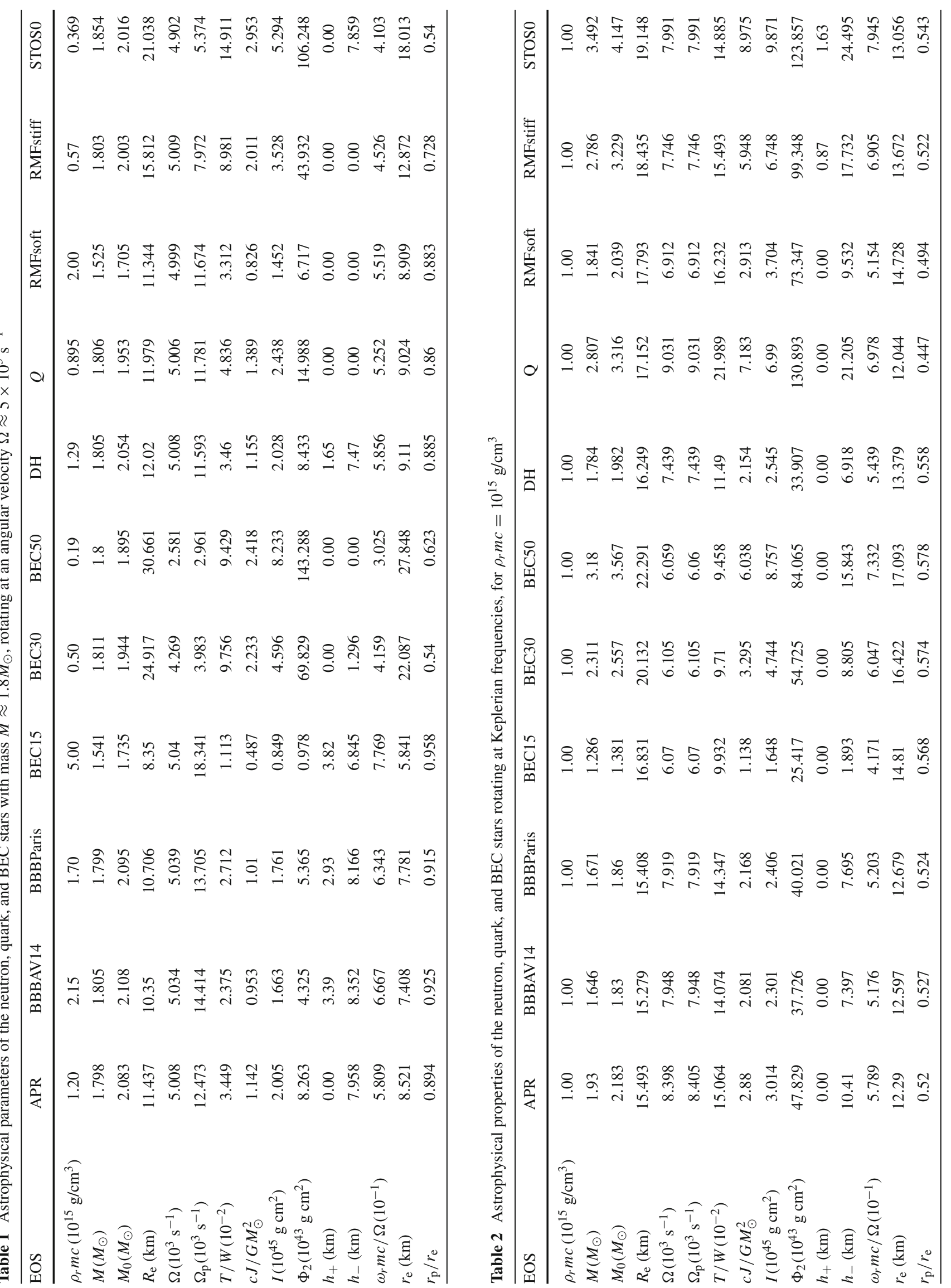


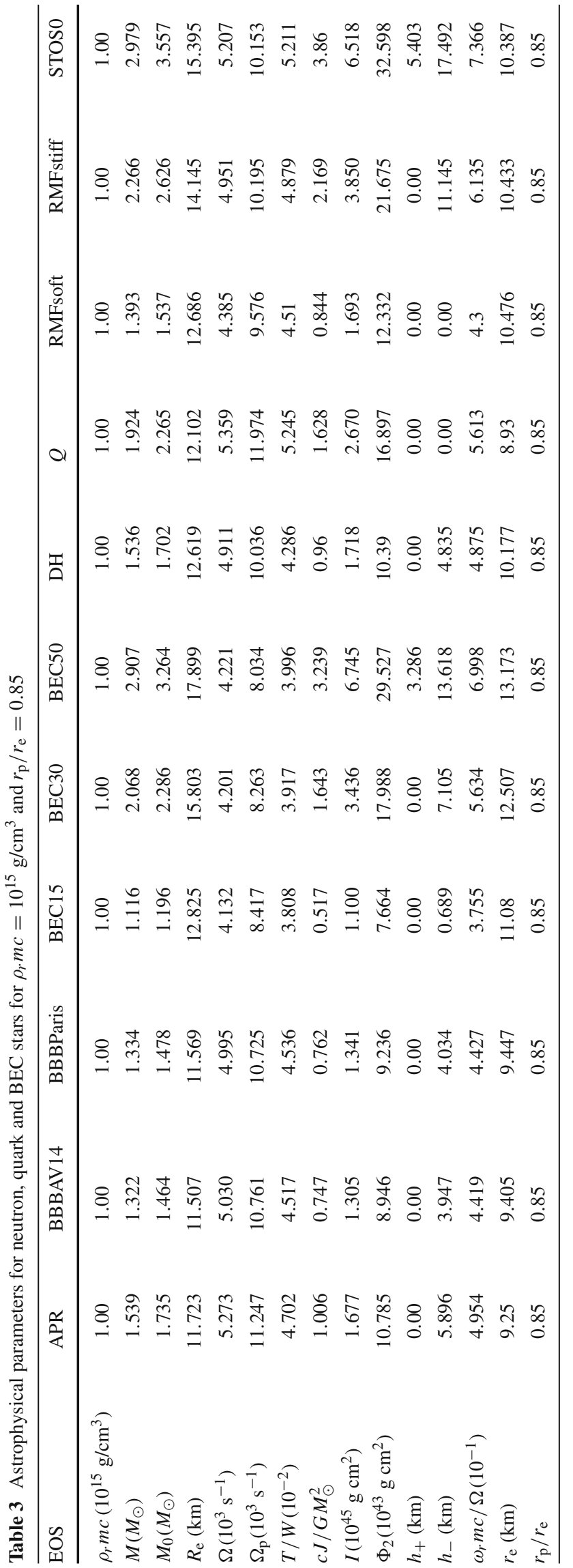

with a numerical value of around $2.9 M_{\odot}$. The lowest mass value is obtained for the BEC15 EOS, $M=1.11 M_{\odot}$. The mass of the BEC30 EOS model, $M=2.068 M_{\odot}$ is significantly smaller than the masses obtained for the Q EOS, with $M=1.924 M_{\odot}$, and for the RMFstiff EOS, having a corresponding mass of $M=2.266 M_{\odot}$. The BEC stars have the larger equatorial radii, of the order of $13-18 \mathrm{~km}$, while the DH EOS has an equatorial radius $r_{\mathrm{e}} \approx 12.6 \mathrm{~km}$, the largest equatorial radius in this class of stellar models. The BEC15 EOS has the smallest moment of inertia and angular momentum per unit mass, with the highest values of these parameters obtained for STOSO EOS. However, the angular momentum per unit mass of the BEC50 EOS just slightly exceeds the angular momentum per unit mass of the RMSstiff EOS, but it is smaller than the $c J / G M$ value of the quark EOS Q.

\section{Electromagnetic and thermodynamic signatures of accretion disks around Bose-Einstein condensate stars}

In the present section we consider the electromagnetic signatures of the accretion disks around neutron, quark, and BEC stars. We consider a comparative study involving three distinct classes of stellar models. The first model corresponds to accretion disks formed around compact general relativistic objects with fixed masses, of the order of $M \approx 1.8 M_{\odot}$, rotating at an angular speed of $\Omega \approx 5 \times 10^{3} \mathrm{~s}^{-1}$. The second case corresponds to accretion disks formed around stars rotating at the maximal Keplerian frequency. Finally, we also consider a third class of models, in which the accretion disk is located around a star with fixed central density and polar to equatorial radius ratio. For all these three cases we consider the disk emissivity properties, which are strongly dependent of the EOS of the dense matter inside the star.

5.1 Electromagnetic spectrum from accretion disks around rotating neutron, quark, and BEC stars with fixed mass and angular velocity

We begin our analysis of the electromagnetic signatures of accretion disks around compact general relativistic objects by considering the case of stars with fixed mass, $M \approx 1.8 M_{\odot}$, and angular velocity of $\Omega \approx 5 \times 10^{3} \mathrm{~s}^{-1}$. The variations of the electromagnetic flux, disk temperature, and luminosity for neutron, quark, and BEC stars with fixed mass and angular velocity are presented in Figs. 4, 5 and 6.

As one can see from the figures, the BEC stars form a distinct group with respect to the neutron and quark stars included in the study. The flux emitted by the accretion disks, presented in Fig. 4, is the smallest for the BEC50 and BEC30 equations of state, respectively. The inner disk edge for the BEC50 and BEC30 EOSs is located at $r / M \simeq 12$ and 


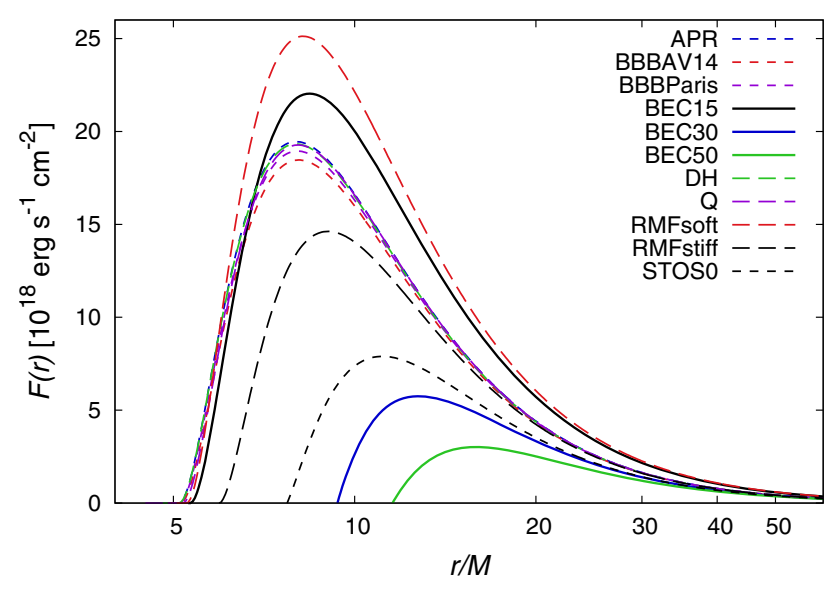

Fig. 4 Electromagnetic fluxes from accretion disks gravitating around compact general relativistic objects, having different equations of state, with mass $M=1.8 M_{\odot}$, and rotating at an angular velocity of $\Omega=$ $5 \times 10^{3} \mathrm{~s}^{-1}$

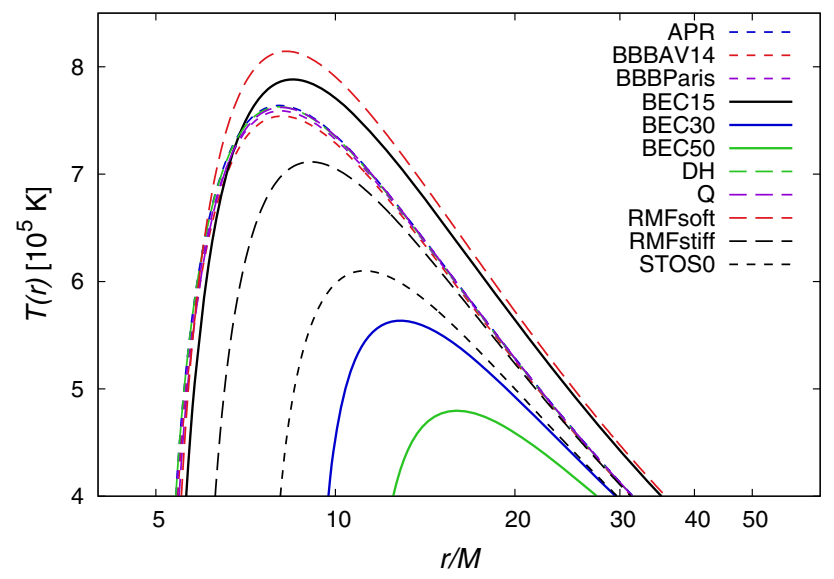

Fig. 5 Temperature distribution of the accretion disks around compact general relativistic objects, having different equations of state, with mass $M=1.8 M_{\odot}$, and rotating at an angular velocity of $\Omega=5 \times$ $10^{3} \mathrm{~s}^{-1}$

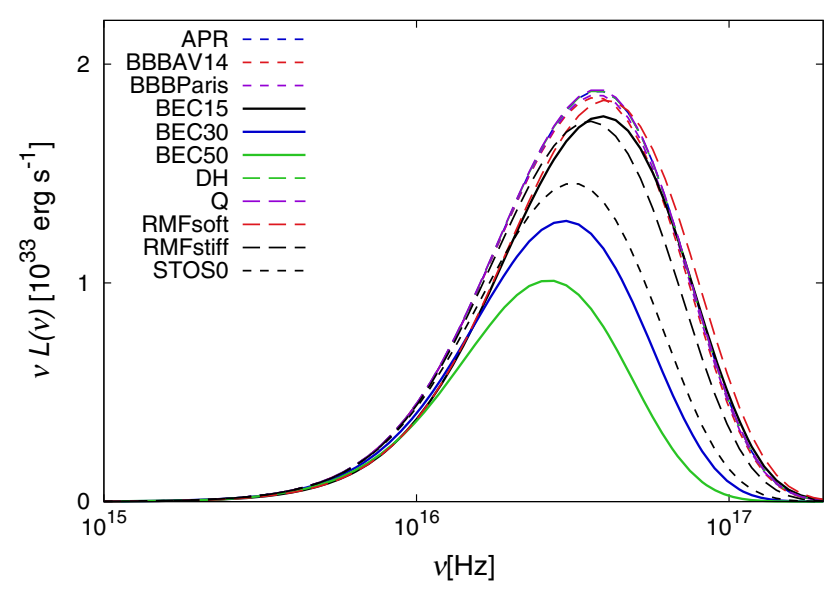

Fig. 6 Luminosity of the accretion disks around compact general relativistic objects, having different equations of state, with mass $M=$ $1.8 M_{\odot}$, and rotating at an angular velocity of $\Omega=5 \times 10^{3} \mathrm{~s}^{-1}$ $r / M \simeq 9$, respectively, at the highest distance from the central object for all considered stars. The maximum value of the flux is obtained for EOS RMFsoft, and this maximum flux value is about twelve and five times bigger than the maximum flux values from the BEC50 and BEC30 EOSs. The flux emitted by the star with EOS BEC15 has high values of the flux, comparable with those from EOS RMFsoft, but this can be explained by the lower mass of the star, $1.541 M_{\odot}$, and the corresponding scaling of the flux. However, for the BEC15 EOS the inner edge of the disk is located at a distance of around $5.5 \times r / M$ from the central object.

The temperature distribution in the disk, shown in Fig. 5, generally follows the same distribution as for the flux profiles, with the BEC stars having some specific distinctive features. The lowest maximum disk temperature is obtained for the BEC50 and BEC30 EOSs, with the maximum temperature located at around $17 \times r / M$ and $13 \times r / M$, respectively. For most of the neutron and quark stars the maximum disk temperature is reached at $r / M \approx 8$. Some specific distinctive features also appear for EOS STOS0, with a maximum temperature located at around $11 \times r / M$, relatively close to the temperature maximum for EOS BEC 30. The highest temperature of the disk is reached by EOS RMFsoft.

The maximum value of the luminosity $v L(v)$ of the disk, presented in Fig. 6, is blue-shifted for the BEC50 and BEC30 EOSs, and it is reached at a smaller frequency. The maximum values of the luminosity are smaller for these BEC EOSs by a factor of around 1.8. Hence a shift in the position of the luminosity maximum, and a lower value of the luminosity give two specific signatures that could help identify BEC50 and BEC30 stars via the study of the luminosity of accretion disks around stars with known physical parameters.

\subsection{Electromagnetic signatures of accretion disks gravitating around neutron, quark, and BEC stars at Keplerian frequencies}

As a second example of specific electromagnetic signatures from accretion disks around neutron, quark, and BEC stars we consider the case of accretion disks formed around compact stars rotating at Keplerian frequencies. The corresponding electromagnetic fluxes, the disk temperature distribution, and the luminosities are presented in Figs. 7, 8, and 9.

From the point of view of the maximum of the flux emission from accretion disks around neutron, quark, and BEC stars rotating at Keplerian frequencies, the stellar models considered in the present study can be roughly divided in two classes: disks with high flux values, and disks with low flux values. As can be seen from Fig. 7, EOSs APR, BBBAV14, $Q$, and $\mathrm{DH}$ have relatively similar maximum flux values, which exceeds the maximum flux values of EOSs RMFstiff, RMFsoft, STOS0, and BEC50, BEC30, and BEC15, respectively. Hence from the point of view of the flux maximum 


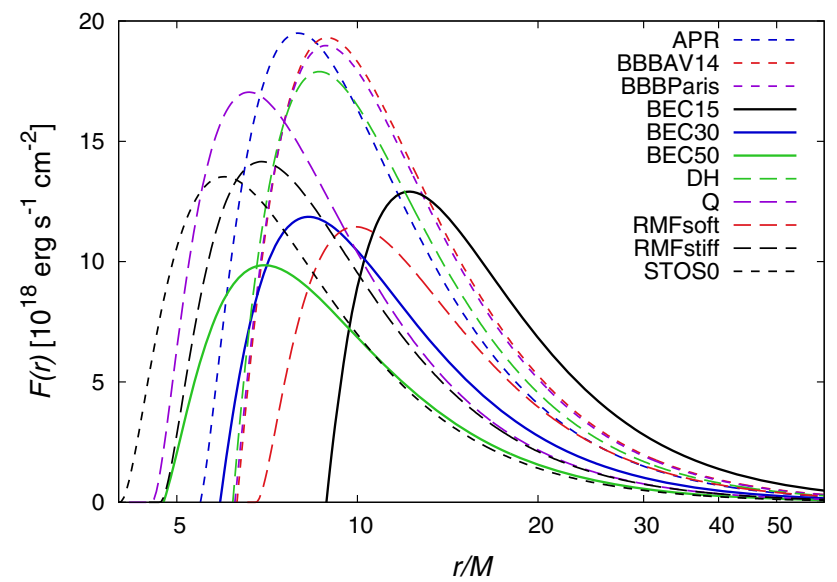

Fig. 7 Electromagnetic fluxes from accretion disks around compact general relativistic objects with different equations state rotating at Keplerian frequencies

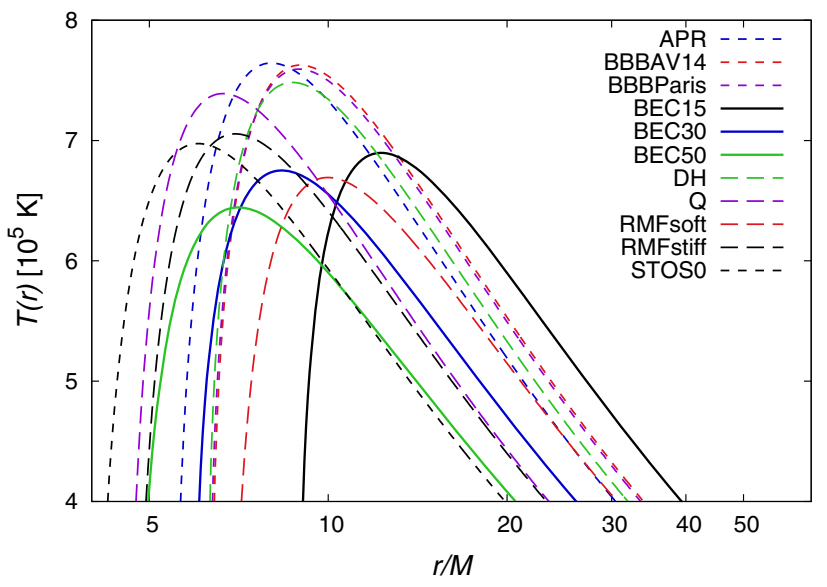

Fig. 8 Temperature distribution of the accretion disks around compact general relativistic objects with different equations of state rotating at Keplerian frequencies

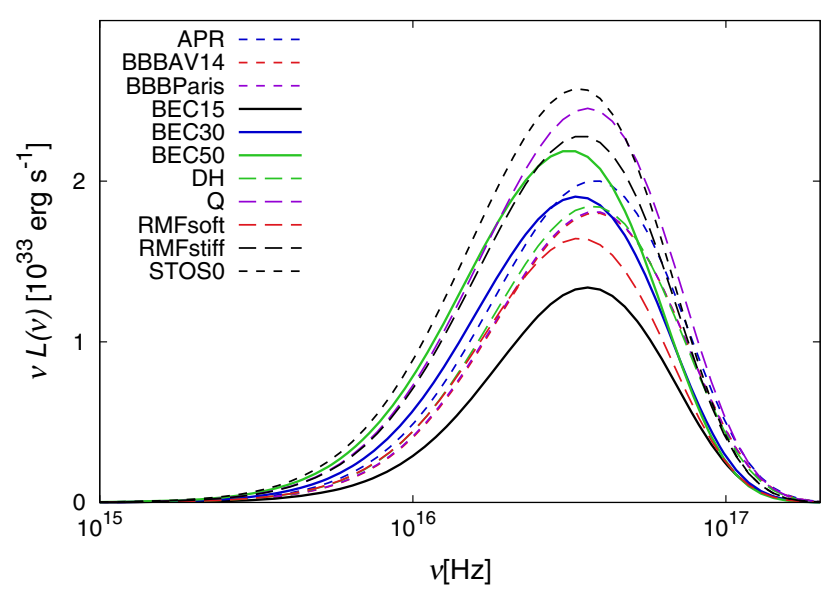

Fig. 9 Luminosity of the accretion disks around compact general relativistic objects with different equations of state rotating at Keplerian frequencies the BEC equations of state present some similarities with the RMFstiff, RMFsoft and STOS0 equations of state. However, important differences do appear in the localization of the position of the maximum of the flux. While for most of the equations of state the maximum is located in the region $7-$ $10 \times r / M$, the position of the maximum flux for EOS BEC15 is at around $13 \times r / M$. For this EOS the inner edge of the disk is located at $9 \times r / M$, while the inner edges of the BEC50 and BEC30 EOSs are located in positions similar to the other neutron and quark matter equations of state. Hence the determination of the position of the flux maximum from an accretion disk could give a clear indication about the nature of the central compact object. The temperature distribution of the disks around stars rotating at Keplerian frequencies, shown in Fig. 8, also indicates the existences of two distinct types of stars, with the BEC stars belonging to the lower temperature group. However, the position of the temperature maximum gives a very clear signature of the nature of the EOS of the compact object. The position of the temperature maximum shifts toward higher distances from the central object with the decrease of the parameter $a / m^{3}$.

The luminosity of the disk, plotted in Fig. 9, shows that the maximum of the luminosity is reached at about the same frequency for all considered equations of state. However, the luminosity maximum has different values for different EOSs, with the BEC15 EOS having the smallest value. The BEC50 and BEC30 EOSs have similar luminosity values as for the other considered neutron and quark matter equations of state.

\subsection{Electromagnetic signatures of accretion disks around} neutron, quark, and BEC stars with fixed central density and $r_{\mathrm{p}} / r_{\mathrm{e}}=0.85$

Finally, we consider the electromagnetic properties of the accretion disks around neutron, quark, and BEC stars with fixed central density and ratio of the polar to the equatorial radius $r_{\mathrm{p}} / r_{\mathrm{e}}=0.85$. All the stellar models have high angular speeds $\Omega$. The variation with respect to $r / M$ of the emitted fluxes, the temperature distribution of the accretion disks, and their luminosities are presented in Figs. 10, 11, and 12.

From the point of view of the electromagnetic flux distribution, presented in Fig. 10, the fluxes can be classified in three groups, having high flux values (EOSs Q and APR), medium values (EOSs RMFstiff, RMFsoft, STOS0, DH, BBBAV14, BBBParis), and low flux values, with all the BEC EOSs belonging to this latter group. It is interesting to note that for all BEC EOSs the maximum value of the flux has (approximately) the same value. However, these maxima are located at different $r / M$, with the maximum of the BEC15 EOS positioned at around $15 \times r / M$ from the central object. The maximum value of the flux of the BEC stars is around three to four times lower than from the stars in the first group, with maximum fluxes (EOSs Q and APR), and 


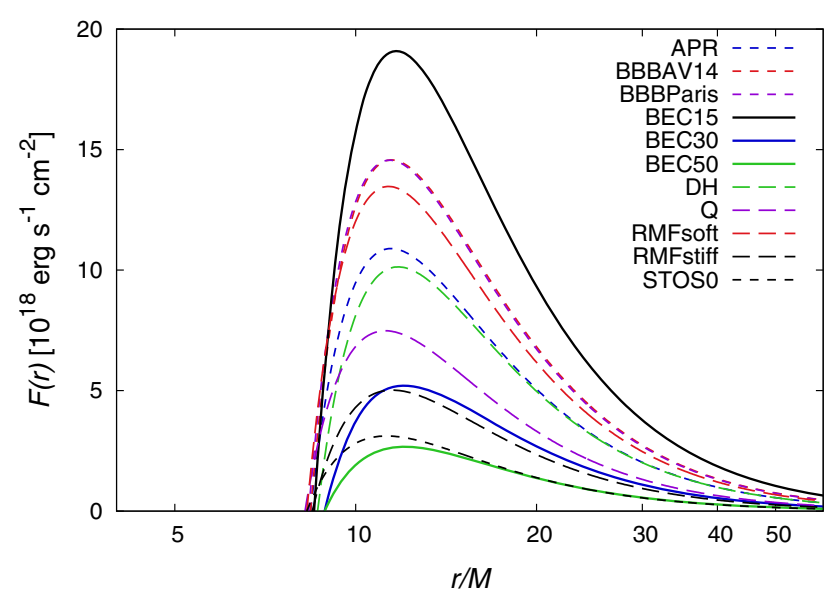

Fig. 10 Electromagnetic fluxes from accretion disks around compact general relativistic objects with fixed central density $\rho_{c}=10^{15} \mathrm{~g} / \mathrm{cm}^{3}$ and $r_{\mathrm{p}} / r_{\mathrm{e}}=0.85$

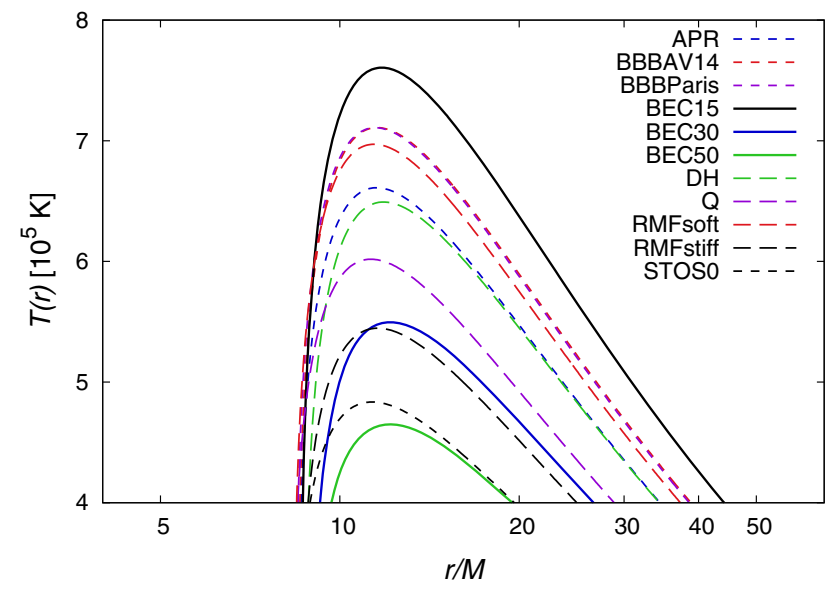

Fig. 11 Temperature distribution of the accretion disks around compact general relativistic objects with fixed central density $\rho_{c}=10^{15}$ $\mathrm{g} / \mathrm{cm}^{3}$ and $r_{\mathrm{p}} / r_{\mathrm{e}}=0.85$

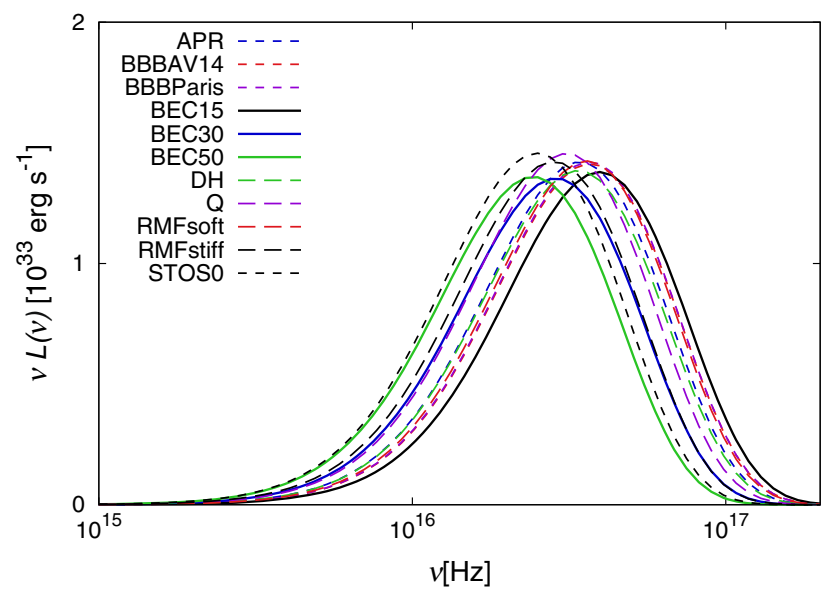

Fig. 12 Luminosity of the accretion disks around compact general relativistic objects with fixed central density $\rho_{c}=10^{15} \mathrm{~g} / \mathrm{cm}^{3}$ and $r_{\mathrm{p}} / r_{\mathrm{e}}=0.85$ around two times smaller as compared to the maximum flux values for the EOSs of the second group. The temperature distribution, shown in Fig. 11, follows the same pattern as the flux, with the maximum of the disk temperature situated at around $18 \times r / M$ for EOS BEC15. The maximum temperature value is very similar for all three BEC equations of state. The luminosities of the disks, plotted in Fig. 12, shows that the maximum of the function $v L(v)$ is reached at roughly the same frequency for all considered EOSs. However, there is a clear theoretical difference in the absolute value of the luminosity maximum, with the BEC15 EOS having the smallest luminosity. Even though EOSs BEC50 and BEC30 have disk luminosities comparable with some neutron star models, they still belong to the class of low luminosity disks.

\subsection{Efficiency of radiation emission from accretion disks} around neutron, quark, and BEC stars

An important observable physical parameter of the disk, which could help to observationally distinguish between different classes of neutron, quark, and BEC stars, is the efficiency $\epsilon$ of the conversion of the accreting mass into radiation, given by Eq. (41). The numerical values of $\epsilon$ show the efficiency of the energy generating mechanism of the mass accretion [97]. From a physical point of view, the binding energy $\widetilde{E}_{\mathrm{ms}}$, or $\widetilde{E}_{\mathrm{e}}$, represents the amount of energy released by the matter leaving the marginally stable orbit, or the inner edge of the disk, touching the surface of the star, and being transferred to the star. The radii of the inner disk edges for different EOS and the efficiency of the radiation emission are presented, for the three stellar models considered in the present paper, in Table 4.

The first two lines of Table 4 contain the conversion efficiency of the compact neutron, quark, and BEC stars rotating at Keplerian frequencies. In this case the values of $\epsilon$ for the BEC stars are in the range of 5-8\%, having values comparable to the $\epsilon$ values for neutron and quark stars. The highest efficiency, $9 \%$, is obtained for the STOSO EOS, while the BEC50 EOS has a conversion efficiency close to that of the quark stars. The smallest $\epsilon$ value is obtained for the BEC15 EOS, showing that these stars are less efficient engines for the conversion of the accreted mass into outgoing radiation.

The values of $\epsilon$ for the compact general relativistic objects with fixed central density and $r_{\mathrm{p}} / r_{\mathrm{e}}$ are given in the second two lines of Table 4 . The values of $\epsilon$ are slightly lower, as compared to the Keplerian rotation case, with the highest value for $\epsilon$ obtained for the STOSO EOS. The smallest value of $\epsilon$ is found for the BEC15 EOS. However, in this case the efficiency of the BEC50 EOS is significantly smaller than the efficiency of the Q EOS, being slightly higher than $\epsilon$ for APR EOS.

The last two lines in Table 4 show the numerical values of $\epsilon$ for disks around compact objects with mass $M=1.8 M_{\odot}$, 


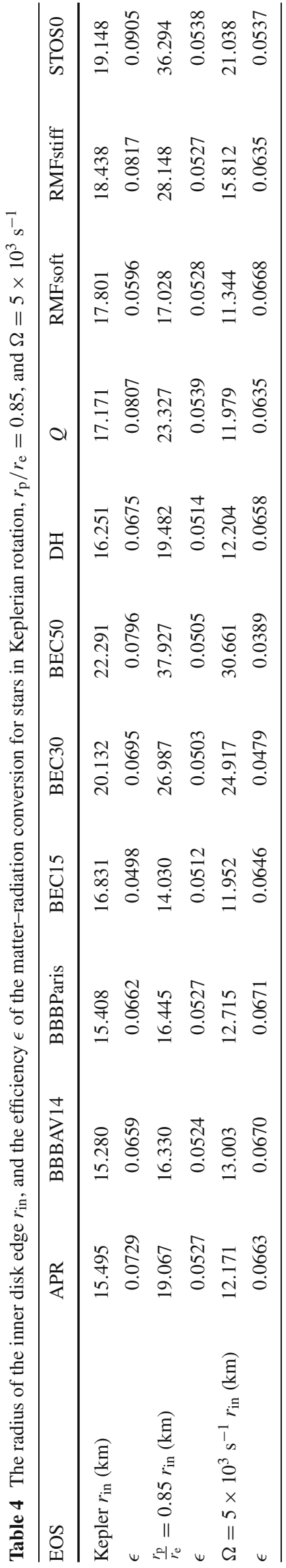

rotating with an angular velocity of $\Omega \approx 5 \times 10^{3} \mathrm{~s}^{-1}$. While the efficiency of the radiation conversion is almost the same for EOSs APR, BBBAV14, BBBParis, DH, Q, RMFsoft, and RMFstiff, it has some lower values for the BEC15 EOS, and much lower values for EOSs BEC30 and BEC50. Relatively low values have also been obtained for the STOSO EOS. The efficiencies of $3.89 \%$ obtained for the BEC15 and BEC50 stars are the lowest accretion disk efficiencies obtained in the present study. The inner edges of the accretion disks around BEC stars are located at a much bigger distance from the central object as compared to the other classes of neutron and quark stars, reaching a value of $31 \mathrm{~km}$ for the BEC50 EOS. Such a far away located inner edge does explain the low efficiency of the corresponding accretion disk.

\section{Discussions and final remarks}

The possible existence of some forms of BECs in compact general relativistic objects, or the existence of pure BEC stars, represents an intriguing, and interesting, possibility, for which a lot of theoretical evidence has been provided. From an observational point of view the most important differences between standard neutron or quark stars are represented by the differences in mass and radius. If the masses and radii of the compact general relativistic object could be measured with high accuracy, these measurements would put very strong direct constraints on the EOS of the dense star. However, presently, there are very few precise determinations of both the mass and the radius of a compact object. Therefore, in the present paper we have proposed, and preliminary investigated, an alternative indirect method, which could help observationally distinguishing between different classes of compact objects, and their equations of state. This method is based on the information extracted from observations of the basic physical properties of matter forming thin accretion disks around rapidly rotating neutron, quark, and BEC stars.

Due to the presence of a strong gravitational field, all the astrophysical quantities related to the observable properties of the accretion disks are dependent, and they can be obtained from the metric of the central compact object [97]. Due to the major differences in the exterior spacetime geometry, neutron, quark, and BEC stars show, at least on the theoretical level, some very important distinct signatures with respect to the disk properties. Therefore, the observational procedure of the analysis of the electromagnetic radiation of accretion disks may allow one to discriminate between neutron, quark, and BEC stars, by giving some specific distinct signatures that could differentiate between compact objects described by different equations of state. In the present paper we have obtained the physical parameters of the disk-effective potential, flux, and emission spectrum 
profiles - for several equations of state of the neutron, quark, and BEC matter, respectively.

As one can see from the flux integral in Eq. (36), as well as from the explicit expressions of the specific energy, specific angular momentum and angular velocity, given by Eqs. (25)(27), respectively, the rather different characteristics of the radial flux distribution over the accretion disk, the disk spectra, and the conversion efficiency are due to the important differences between the metric potentials of the neutron, quark, and BEC stars, respectively. Even if the total mass and the angular velocity are the same for each type of the rotating central object (neutron, quark or BEC star), with the stars having similar values of $\Omega, \widetilde{E}$ and $\widetilde{L}$, the radiation properties of the accretion disks around these compact general relativistic objects exhibit observable differences [97]. The physical reason for these differences is that the proper volume, and in turn the function $\sqrt{-g}$, used in the calculation of the flux integral, is strongly dependent on the behavior of the metric component $g_{r r}=(\partial \bar{r} / \partial r)^{2} g_{\overline{r r}}$, and therefore on the geometry of the space-time. The latter expression contains the derivatives with respect to the radial coordinate $r$ of the metric components $\rho(r)$ and $\gamma(r)$, respectively, via the coordinate transformation between the coordinates $\bar{r}$ and $r$, which are extremely sensitive to the slope of the functions $\rho(r)$ and $\gamma(r)$ [97]. Therefore, although the inner edges of the disks are located at almost the same radii, the maximum amplitudes and the numerical values of the energy fluxes emerging form the disk surface, and propagating in any solid angle, may show considerable differences for different equations of state of the neutron, quark, and BEC matter. These essentially geometrical effects also give rise to the distinctive features in the disk spectra for the various types of central stars.

In our preliminary and idealized theoretical study of the accretion disk properties around BEC stars we have found a number of observational signatures distinguishing this class of stars from the neutron and quark stars. These specific properties are the distinct positions of the maxima of the flux, of the temperature distribution, and of the luminosity of the disk, the position of the inner edge of the disk, and the radiation efficiency conversion. In all three different classes of rotating stars we have analyzed these signatures do appear distinctly. Moreover, BEC stars have a mass-radius relation that can also help in discriminating them with respect to other classes of neutron and quark stars.

In the present paper, which represents a first step in the investigation of the complex astrophysical problem of the radiation emission from accretion disks around compact objects with different nuclear equations of state, we made the fundamental assumption that the inner edge of the disk is located at the ISCO radius. This means that the ISCO position is only determined by the space-time geometry around the compact object. However, a number of important phys- ical factors can affect the position and location of the inner edge of the disk. For example, in the case of neutron stars, the inner edge of the disk is usually set by the magnetosphere around the star, and it is not determined by the metric of the space-time only. But, in order to simplify the discussion of the complex physics of the accretion disks, and by taking into account the preliminary and purely theoretical nature of this study, in the present paper we consider only an ideal case, which allows us to start our analysis from very simple physical and astrophysical considerations. As already mentioned, an important physical parameter that strongly influences the ISCO position is the magnetic field of the compact central object, and so is its magnetosphere. The magnetic field lines originating from the central object can have a considerable effect on the evolution of the accretion processes and on the disk structure. The effects of magnetic fields on the accretion processes in a compact, spherically symmetric geometry of Schwarzschild type (which describes the exterior geometry for both black holes and compact stars) were considered in [131]. The magnetic field was assumed to be asymptotically uniform, and axisymmetric tidal structures were also taken into account. Due to the presence of the magnetic field and of the tidal perturbations, the accretion disk shrinks in size, and the marginally stable orbits shift toward the central object. On the other hand the presence of the magnetic perturbation leads to an increase in the disk radiation intensity from the accretion disk. However, the position of the maximum of the radiation does not change, and the radiation spectrum is blue-shifted. Interestingly, the conversion efficiency decreases due to the presence of the magnetic fields and of the tidal perturbations. Alternative studies of the effects of the neutron star's magnetic field on the exterior metric and the position of ISCO's can be found in $[132,133]$.

In the present paper we have proposed a method for discriminating between different types of compact objects that was proposed, and observationally tested, in the case of the black holes. Black holes have an event horizon (or a very special surface with similar properties) that prevent them from emitting any form of electromagnetic radiation [134]. This makes the radiation emission from the disk, and due to accretion processes, the dominant electromagnetic energy emission mechanism, thus allowing the possibility of determining the central black hole properties from the radiation spectrum of the disk, without any possible interference of the electromagnetic signals from the black hole. On the other hand, neutron stars have a solid radiation emitting surface (crust), which makes it very difficult to distinguish between the radiation of the star and of the disk itself. That is why the continuum-fitting method was not applied for the study of neutron stars [134], and up to now there is no published observational study of the possibility of testing the neutron star EOS by using the electromagnetic spectrum of a thin 
disk. On the other hand we would like to point out that the electromagnetic emissivity of the zero temperature gravitationally bounded BEC matter, in which all particles are in the quantum ground state, described by a single wave function, is very low [135]. This is because the effective plasma frequency of the condensed mater is very high. Therefore we may assume that the radiation emissivity of the BEC stars is very low, and they are very "black". Consequently, the electromagnetic emissivity of the BEC star-disk system may be dominated by the disk emission. This situation is similar to the case of quark stars. Since quark matter has a very high plasma frequency, $\omega_{\mathrm{p}}$, the photon emissivity of strange quark stars is very low $[136,137]$. This is due to the fact that the propagation of electromagnetic waves having frequencies lower than $\omega_{\mathrm{p}}$ is exponentially damped. Hence, only photons produced just below a few fermi from the surface, with outwards pointing momenta, can be emitted by the strange star. Hence the equilibrium photon emissivity from a strange star is negligible small, as compared to the black body one. Moreover, the spectrum of the emitted equilibrium photons is very hard, with $\hbar \omega>20 \mathrm{MeV}$ [136]. Of course, BEC stars, as well as the quark stars, may have a crust (solid surface), representing a powerful source of electromagnetic radiation. The presence of such a crust would further complicate the possibility of discriminating between BEC stars and the other types of compact general relativistic objects.

Another important point we would like to stress is that from an observational point of view not all the parameters of the BEC star-disk system can be determined independently from astrophysical observations. This means that a degeneracy between the EOS and the nature of the compact object, and the values of some physical parameters of the model, could always exist. A full test of the BEC EOS would require the precise knowledge of the mass, radius, and spin of the central object, as well as all the electromagnetic disk properties. In this ideal case, extremely difficult to achieve from an observational point of view, the fitting of the observational data could provide a convincing test of the nature of the central object. Probably in the near future no such increase in the precision of the astrophysical observations will be achieved, thus rendering the direct determination of the EOS of the nuclear or condensed matter beyond the present observational capabilities. On the other hand one could expect an increase in the determination of the masses and radii for various compact objects, and these measurements may give some hints on the true nature of the EOS of the dense matter.

In conclusion, once the precision of the astrophysical data is drastically increased, the observational study of the thin accretion disks around rapidly rotating compact objects, and of their electromagnetic properties (flux, temperature distribution and luminosity), may provide a powerful tool in distinguishing between different classes of dense stellar objects, as well as for discriminating between the different equations of state of the dense matter.

Acknowledgments We would like to thank the anonymous referee for comments and suggestions that helped us to significantly improve our manuscript. The authors are very grateful to Dr. Gabriela Mocanu for her constant support, help, and useful comments. BD acknowledges the support of "Babes-Bolyai" University Cluj-Napoca through a Research Excellence scholarship. This work is partially supported by a grant of the Romanian National Authority of Scientific Research, Program for research-Space Technology and Advanced Research-STAR, Project No. 72/29.11.2013.

Open Access This article is distributed under the terms of the Creative Commons Attribution 4.0 International License (http://creativecomm ons.org/licenses/by/4.0/), which permits unrestricted use, distribution, and reproduction in any medium, provided you give appropriate credit to the original author(s) and the source, provide a link to the Creative Commons license, and indicate if changes were made.

Funded by SCOAP 3 .

\section{References}

1. S.N. Bose, Z. Phys. 26, 178 (1924)

2. A. Einstein, Sitzungsberichte der Preussischen Akademie der Wissenschaften. Phys.-Math. Kl. 1924, 261 (1924)

3. A. Einstein, Sitzungsberichte der Preussischen Akademie der Wissenschaften. Phys.-Math. K1. 1925, 3 (1925)

4. F. Dalfovo, S. Giorgini, L.P. Pitaevskii, S. Stringari, Rev. Mod. Phys. 71, 463 (1999)

5. E.A. Cornell, C.E. Wieman, Rev. Mod. Phys. 74, 875 (2002)

6. W. Ketterle, Rev. Mod. Phys. 74, 1131 (2002)

7. R.A. Duine, H.T.C. Stoof, Phys. Rep. 396, 115 (2004)

8. Q. Chen, J. Stajic, S. Tan, K. Levin, Phys. Rep. 412, 1 (2005)

9. L. Pitaevskii, S. Stringari, Bose-Einstein Condensation (Clarendon Press, Oxford, 2003)

10. C.J. Pethick, H. Smith, Bose-Einstein Condensation in Dilute Gases (Cambridge University Press, Cambridge, 2008)

11. A. Griffin, T. Nikuni, E. Zaremba, Bose-Condensed Gases at Finite Temperatures (Cambridge University Press, Cambridge, 2009)

12. M.H. Anderson, J.R. Ensher, M.R. Matthews, C.E. Wieman, E.A. Cornell, Science 269, 198 (1995)

13. C.C. Bradley, C.A. Sackett, J.J. Tollett, R.G. Hulet, Phys. Rev. Lett. 75, 1687 (1995)

14. K.B. Davis, M.O. Mewes, M.R. Andrews, N.J. van Drutten, D.S. Durfee, D.M. Kurn, W. Ketterle, Phys. Rev. Lett. 75, 3969 (1995)

15. S.J. Sin, Phys. Rev. D 50, 3650 (1994)

16. S.U. Ji, S.J. Sin, Phys. Rev. D 50, 3655 (1994)

17. W. Hu, R. Barkana, A. Gruzinov, Phys. Rev. Lett. 85, 1158 (2000)

18. J. Goodman, New Astron. 5, 103 (2000)

19. P.J.E. Peebles, Astrophys. J. 534, L127 (2000)

20. A. Arbey, J. Lesgourgues, P. Salati, Phys. Rev. D 68, 023511 (2003)

21. C.G. Boehmer, T. Harko, JCAP 06, 025 (2007)

22. J.-W. Lee, Phys. Lett. B 681, 118 (2009)

23. J.-W. Lee, S. Lim, JCAP 1001, 007 (2010)

24. T. Harko, JCAP 1105, 022 (2011)

25. V.H. Robles, T. Matos, Mon. Not. R. Astron. Soc. 422, 282 (2012)

26. M. Dwornik, Z. Keresztes, L.A. Gergely, in Chapter 6 of "Recent Development in Dark Matter Research", eds. by N. Kinjo, A. Nakajima (Nova Science Publishers, 2014). arXiv:1312.3715 
27. F.S. Guzman, F.D. Lora-Clavijo, J.J. Gonzalez-Aviles, F.J. RiveraPaleo (2013). arXiv: 1310.3909

28. T. Harko, E.J.M. Madarassy, JCAP 01, 020 (2012)

29. B. Kain, H.Y. Ling, Phys. Rev. D 82, 064042 (2010)

30. N.T. Zinner, Phys. Res. Int. 2011, 734543 (2011)

31. P.-H. Chavanis, Phys. Rev. D 84, 043531 (2011)

32. P.-H. Chavanis, L. Delfini, Phys. Rev. D 84, 043532 (2011)

33. P.-H. Chavanis, Phys. Rev. D 84, 063518 (2011)

34. P.-H. Chavanis, Phys. Rev. E 84, 031101 (2011)

35. T. Rindler-Daller, P.R. Shapiro, Mon. Not. R. Astron. Soc. 422, 135 (2012)

36. T. Rindler-Daller, P.R. Shapiro (2013). arXiv:1312.1734

37. M.O.C. Pires, J.C.C. de Souza, JCAP 11, 024 (2012)

38. T. Harko, Phys. Rev. D 83, 123515 (2011)

39. T. Harko, G. Mocanu, Phys. Rev. D 85, 084012 (2012)

40. T. Harko, Mon. Not. R. Astron. Soc. 413, 3095 (2011)

41. P.-H. Chavanis, Astron. Astrophys. 537, A127 (2012)

42. R.C. Freitas, S.V.B. Goncalves, JCAP 04, 049 (2013)

43. H. Velten, E. Wamba, Phys. Lett. B 709, 1 (2012)

44. E.J.M. Madarassy, V.T. Toth, Comput. Phys. Commun. 184, 1339 (2013)

45. F.S. Guzman, F.D. Lora-Clavijo, J.J. Gonzalez-Aviles, F.J. RiveraPaleo, JCAP 09, 034 (2013)

46. J.C.C. de Souza, M.O.C. Pires (2014). arXiv: 1401.6142

47. V.T. Toth (2014). arXiv: 1402.0600

48. T. Harko, Phys. Rev. D 89, 084040 (2014)

49. M.-H. Li, Z.-B. Li, Phys. Rev. D 89, 103512 (2014)

50. T. Harko, M.J. Lake, Phys. Rev. D 91, 045012 (2015)

51. M.G. Alford, K. Rajagopal, T. Schaefer, A. Schmitt, Rev. Mod. Phys. 80, 1455-1515 (2008)

52. M. Randeria, E. Taylor, Ann. Rev. Condens. Matter Phys. 5, 209 (2014)

53. M. Baldo, U. Lombardo, P. Schuck, Phys. Rev. C 52, 975 (1995)

54. H. Stein, A. Schnell, T. Alm, G. Ropke, Z. Phys. A 351, 295 (1995)

55. U. Lombardo, P. Nozieres, P. Schuck, H.-J. Schulze, A. Sedrakian, Phys. Rev. C 64, 064314 (2001)

56. E. Babaev, Phys. Rev. B 63, 184514 (2001)

57. B. Kerbikov, Phys. At. Nucl. 65, 1918 (2002)

58. P. Castorina, G. Nardulli, D. Zappala, Phys. Rev. D 72, 076006 (2005)

59. A.H. Rezaeian, H.J. Pirner, Nucl. Phys. A 779, 197 (2006)

60. J. Deng, A. Schmitt, Q. Wang, Phys. Rev. D 76, 034013 (2007)

61. T. Brauner, Phys. Rev. D 77, 096006 (2008)

62. M. Matsuzaki, Phys. Rev. D 82, 016005 (2010)

63. H. Abuki, G. Baym, T. Hatsuda, N. Yamamoto, Phys. Rev. D 81, 125010 (2010)

64. Y. Nishida, H. Abuki, Phys. Rev. D 72, 096004 (2005)

65. G. Sun, L. He, P. Zhuang, Phys. Rev. D 75, 096004 (2007)

66. L. He, P. Zhuang, Phys. Rev. D 75, 096003 (2007)

67. L. He, P. Zhuang, Phys. Rev. D 76, 056003 (2007)

68. L. He, Phys. Rev. D 82, 096003 (2010)

69. L. He, S. Mao, P. Zhuang, Int. J. Mod. Phys. A 28, 1330054 (2013)

70. N.K. Glendenning, Compact Stars, Nuclear Physics, Particle Physics and General Relativity (Springer, New York, 2000)

71. S. Banik, D. Bandyopadhyay, Phys. Rev. D 67, 123003 (2003)

72. S. Banik, M. Hanauske, D. Bandyopadhyay, W. Greiner, Phys. Rev. D 70, 123004 (2004)

73. S. Weissenborn, D. Chatterjee, J. Schaffner-Bielich, Nucl. Phys. A 881, 62 (2012)

74. S. Weissenborn, D. Chatterjee, J. Schaffner-Bielich, Phys. Rev. C 85, 065802 (2012)

75. T. Sakaia, K. Yazaki, K. Shimizu, Nucl. Phys. A 594, 247 (1995)

76. T. Sakai, K. Shimizu, K. Yazaki, Prog. Theor. Phys. Suppl. 137, $121(2000)$

77. T. Hell, W. Weise, Phys. Rev. C 90, 045801 (2014)
78. J.I. Kapusta, Phys. Rev. D 93, 251801 (2004)

79. H. Abuki, Nucl. Phys. A 791, 117 (2007)

80. P.H. Chavanis, T. Harko, Phys. Rev. D 86, 064011 (2012)

81. X.Y. Li, T. Harko, K.S. Cheng, JCAP 06, 001 (2012)

82. X.Y. Li, F. Wang, K.S. Cheng, JCAP 10, 031 (2012)

83. P.H. Chavanis (2014). arXiv: 1412.0005

84. S. Latifah, A. Sulaksono, T. Mart (2014). arXiv:1412.1556

85. E.J.M. Madarassy, V.T. Toth (2014). arXiv:1412.7152

86. D.N. Page, K.S. Thorne, Astrophys. J. 191, 499 (1974)

87. K.S. Thorne, Astrophys. J. 191, 507 (1974)

88. Z. Kovács, T. Harko, Phys. Rev. D 82, 124047 (2010)

89. T. Harko, Z. Kovács, F.S.N. Lobo, Class. Quantum Gravity 26, 215006 (2009)

90. T. Harko, Z. Kovács, F.S.N. Lobo, Phys. Rev. D 78, 084005 (2008)

91. T. Harko, Z. Kovács, F.S.N. Lobo, Phys. Rev. D 79, 064001 (2009)

92. C.S.J. Pun, Z. Kovács, T. Harko, Phys. Rev. D 78, 024043 (2008)

93. C.S.J. Pun, Z. Kovács, T. Harko, Phys. Rev. D 78, 084015 (2008)

94. T. Harko, Z. Kovács, F.S.N. Lobo, Phys. Rev. D 80, 044021 (2009)

95. T. Harko, Z. Kovács, F.S.N. Lobo, Class. Quantum Gravity 27, 105010 (2010)

96. T. Harko, Z. Kovács, F.S.N. Lobo, Class. Quantum Gravity 28, 165001 (2011)

97. Z. Kovács, K.S. Cheng, T. Harko, Astron. Astrophys. 500, 621 (2009)

98. D. Torres, Nucl. Phys. B 626, 377 (2002)

99. Y.F. Yuan, R. Narayan, M.J. Rees, Astrophys. J. 606, 1112 (2004)

100. Z. Kovács, K.S. Cheng, T. Harko, MNRAS 400, 1632 (2009)

101. G.R. Mocanu, D. Grumiller, Phys. Rev. D 85, 105022 (2012)

102. C. Bambi, Phys. Rev. D 87, 084039 (2013)

103. C. Bambi, D. Malafarina, Phys. Rev. D 88, 064022 (2013)

104. L. Kong, Z. Li, C. Bambi, Astrophys. J. 797, 78 (2014)

105. N. Stergioulas, J.L. Friedman, Astrophys. J. 444, 306 (1995)

106. N. Stergioulas, W. Kluzniak, T. Bulik, Astron. Astrophys. 352, L116 (1999)

107. N. Stergioulas, Living Rev. Relativ. 6, 3 (2003)

108. N.I. Shakura, R.A. Sunyaev, Astron. Astrophys. 24, 33 (1973)

109. I.D. Novikov, K.S. Thorne, in Black Holes, ed. by C. DeWitt, B. DeWitt (Gordon and Breach, New York, 1973)

110. J.P. Luminet, Astron. Astrophys. 75, 228 (1979)

111. S. Bhattacharyya, R. Misra, A.V. Thampan, Astrophys. J. 550, 841 (2001)

112. S. Bhattacharyya, A.V. Thampan, I. Bombaci, Astron. Astrophys. 372, 925 (2001)

113. S.N. Zhang, W. Cui, W. Chen, Astrophys. J. 482, L155 (1997)

114. C. Bambi, E. Barausse, Astrophys. J. 731, 121 (2011)

115. A.K. Kulkarni, R.F. Penna, R.V. Shcherbakov, J.F. Steiner, R. Narayan, A. Sadowski, Y. Zhu, J.E. McClintock, S.W. Davis, J.C. McKinney, Mon. Not. R. Astron. Soc. 414, 1183 (2011)

116. C. Bambi, JCAP 1209, 014 (2012)

117. L. Gou, J.E. McClintock, R.A. Remillard, J.F. Steiner, M.J. Reid, J.A. Orosz, R. Narayan, M. Hanke, J. Garcia, Astrophys. J. 790, 29 (2014)

118. A. Akmal, V.R. Pandharipande, D.G. Ravenhall, Phys. Rev. C 58, 1804 (1998)

119. G. Baym, H.A. Bethe, C.J. Pethick, Nucl. Phys. A 175, 225 (1971)

120. G. Baym, C.J. Pethick, P. Sutherland, Astrophys. J. 170, 299 (1971)

121. R.P. Feynman, N. Metropolis, E. Teller, Phys. Rev. 75, 1561 (1949)

122. F. Douchin, P. Haensel, Astron. Astrophys. 380, 151 (2001)

123. H. Shen, H. Toki, K. Oyamatsu, K. Sumiyoshi, Nucl. Phys. A 637, 435 (1998)

124. S. Kubis, M. Kutschera, Phys. Lett. B 399, 191 (1997)

125. M. Baldo, I. Bombaci, G.F. Burgio, Astron. Astrophys. 328, 274 (1997)

126. N. Itoh, Prog. Theor. Phys. 44, 291 (1970) 
127. A.R. Bodmer, Phys. Rev. D 4, 1601 (1971)

128. E. Witten, Phys. Rev. D 30, 272 (1984)

129. V.V. Usov, T. Harko, K.S. Cheng, Astrophys. J. 620, 915 (2005)

130. M. Shibata, M. Sasaki, Phys. Rev. D 58, 104011 (1998)

131. Z. Kovács, L.A. Gergely, M. Vasúth, Phys. Rev. D 84, 024018 (2011)

132. M. Stute, M. Camenzind, Mon. Not. R. Astron. Soc. 336, 831 (2002)
133. A.F. Gutierrez-Ruiz, C.A. Valenzuela-Toledo, L.A. Pachon, Univ. Sci. 19, 63 (2013)

134. R. Narayan, J.E. McClintock, New Astron. Rev. 51, 733 (2008)

135. S. Giovanazzi, G. Kurizki, I.E. Mazets, S. Stringari, Europhys. Lett. 56, 1 (2001)

136. K.S. Cheng, T. Harko, Astrophys. J. 596, 451 (2003); T. Harko and K. S. Cheng. Astrophys. J. 622, 1033 (2005)

137. T. Harko, K.S. Cheng. Astrophys. J. 622, 1033 (2005) 